\title{
Multilayered spraying and gradient dotting of nanodiamond-polycaprolactone guidance channels for restoration of immune homeostasis
}

\author{
Yun Qian ${ }^{1}$, Yuan Cheng ${ }^{2}$, Yuanming Ouyang ${ }^{1,3}$, Wei-En Yuan ${ }^{2}$ and Cunyi Fan ${ }^{1,3}$
}

\begin{abstract}
The major problem in peripheral nerve repair is restoration of the microenvironment rather than traditional structural reconstruction. Nanodiamonds (NDs), highly biocompatible carbon nanoparticles, are widely applied in medical engineering. They may alleviate inflammatory insults in peripheral nerve injury because they can induce macrophage polarization from a proinflammatory to an anti-inflammatory state. Here we report a concentric multilayered spraying manufacturing process to fabricate microporous ND/polycaprolactone (PCL) nerve bridges. We investigated the proliferative, adhesive, and glioprotective role of these bridges in Schwann cells in vitro. We further evaluated their long-term in vivo performance in a 20-mm sciatic nerve defect rat model. ND/PCL nerve bridges are comparable to autografts in functional, electrophysiological, and morphological sciatic nerve repair. They ameliorate the immune milieu by inducing M1 to M2 macrophage polarization. In addition, they pose no harm to major organs after 4 months of implantation. These findings show the promising roles of ND-based nanotechnology in neuroengineering.
\end{abstract}

\section{Introduction}

Peripheral nerve defects are the most severe kind of injury in the peripheral nervous system. These defects cause life-long disability for hundreds of thousands of people as a global health problem. With the development of microsurgical techniques, better alternatives, such as biosynthetic nerve conduits, are gradually being used for long nerve gaps. These alternatives may replace nerve autografts because they avoid tissue damage to the donor site and mismatch with the desired nerve defect ${ }^{1-3}$. However, their performance is still not satisfactory.

\footnotetext{
Correspondence: Cunyi Fan (cyfan@sjtu.edu.cn)

Wei-En Yuan (yuanweien@sjtu.edu.cn)

${ }^{1}$ Department of Orthopedics, Shanghai Jiao Tong University Affiliated Sixth People's Hospital, 200233 Shanghai, China

${ }^{2}$ Engineering Research Center of Cell \& Therapeutic Antibody, Ministry of Education, and School of Pharmacy, Shanghai Jiao Tong University, 200240 Shanghai, China

Full list of author information is available at the end of the article.

These authors contributed equally: Yun Qian, Wei-En Yuan
}

Carbon-based nanostructured biomaterials can interact with living tissues in a controlled, engineered, and modified manner ${ }^{4}$. They have many allotropes, including carbon nanotubes (CNTs), graphene, and nanodiamonds $(\mathrm{NDs})^{5-7}$. CNT scaffolds can increase axon growth speed and anisotropic structure under electrical stimulation and contact guidance to reinforce structural stability with biodegradable conduit materials ${ }^{8}$. Salehi et al. fabricated polylactic acid/multiwalled $\mathrm{CNT} /$ gelatin conduits to repair 10-mm sciatic nerve defects. Compared with those of the control group, increased compound muscle action potential, sciatic function index, and muscle weight were observed in the CNT-based conduit ${ }^{9}$.

Graphene is a two-dimensional (2D) material characterized by carbon sp2 hybridization and a honeycomb lattice. Their astonishing electric and mechanical properties have enabled graphene and graphene oxide (GO) to become promising materials in neuroengineering. We previously reported integrated multilayer fabrication of

\section{(c) The Author(s) 2019}

(c) (i) Open Access This article is licensed under a Creative Commons Attribution 4.0 International License, which permits use, sharing, adaptation, distribution and reproduction c. in any medium or format, as long as you give appropriate credit to the original author(s) and the source, provide a link to the Creative Commons license, and indicate if changes were made. The images or other third party material in this article are included in the article's Creative Commons license, unless indicated otherwise in a credit line to the material. If material is not included in the article's Creative Commons license and your intended use is not permitted by statutory regulation or exceeds the permitted use, you will need to obtain permission directly from the copyright holder. To view a copy of this license, visit http://creativecommons.org/licenses/by/4.0/. 
polydopamine- and arginylglycylaspartic acid-modified graphene/polycaprolactone (PCL) conduits in the application of long-range sciatic nerve defect regeneration. Conductive graphene-based conduits can greatly increase neural expression and improve axonal regeneration and remyelination ${ }^{10}$. We also discussed GO/PCL conduits via integration molding and evaluated their potential in stimulating angiogenesis to promote sciatic nerve defect repair. The possible mechanism was via vascular endothelial growth factor (VEGF) signaling ${ }^{11}$. Although they can effectively repair nerve injury, graphene-loaded nerve scaffolds are generally not very biocompatible, which limits their biomedical application.

Unlike CNTs and graphene, NDs are not electrically conductive $^{12}$. However, they have excellent biocompatibility and are widely used for surface coating, drug delivery, and biological imaging. The application of $400 \mu \mathrm{g} \mathrm{ml}^{-1} \mathrm{NDs}$ exerted insignificant effects on human embryonic kidney cells $^{13}$. The application of $150 \mu \mathrm{g} \mathrm{ml}^{-1}$ NDs significantly reduced neuroblastoma cell metabolic activity, while $100 \mu \mathrm{g} \mathrm{ml}^{-1}$ was the maximal biocompatible concentration ${ }^{14}$. Compared with CNTs and other carbon materials, NDs have limited cytotoxicity to neuroblastoma cells. They did not induce oxidative stress or affect the mitochondrial membrane potential ${ }^{15}$. Amine-functionalized NDs facilitated neural proliferation, attachment, and differentiation ${ }^{16}$. Nerve cells seeded on different ND-coated sheets extended their protuberances widely. They had performances similar to those of cells cultured on protein-coated biomaterials in terms of cell attachment, neurite outgrowth, and excitability as well as functional electrical activity ${ }^{17}$.

In addition to excellent biocompatibility, NDs might regulate the immune microenvironment by activating M2 (alternatively activated) macrophages and reducing M1 (classically activated) behavior ${ }^{18}$. We wondered whether NDs have similar performance in severe nerve injury. Peripheral nerves begin self-regeneration immediately after injury and are affected by macrophages. There are two types of macrophages, M1 and M2. The former type is proinflammatory and exerts limited toxicity to mammalian cells. The latter type facilitates tissue proliferation and regeneration. Mokarram et al. induced M1 macrophages to the M2 phenotype and found improved regeneration in tibial nerve transection injury ${ }^{19}$. The increase in the M2 type was accompanied by a decrease in the M1 type, indicating macrophage polarization during the nerve regenerative process ${ }^{20}$. Furthermore, we identified the upregulation of signal transducer and activator of transcription-3 (STAT-3) and downregulation of nuclear factor $-\kappa B(N F-\kappa B)$ after ND application.

In this study, we report a concentric multilayered spraying manufacturing process to fabricate microporous ND/PCL nerve bridges. We investigated their proliferative, adhesive, and glioprotective role in Schwann cells (SCs) in vitro. We further evaluated their long-term in vivo performance in a 20-mm sciatic nerve defect rat model. ND/PCL nerve bridges were compared with autografts in functional, electrophysiological, and morphological sciatic nerve repair. We further investigated ND regulation of macrophage phenotypes and their roles in clearing inflammatory substances and restoring immune balance in neuroengineering.

\section{Materials and methods}

\section{Scaffold fabrication and characterization}

NDs were purchased from Nanjing XFNANO Materials Tech Co., Ltd. According to the supplier, the NDs have a purity of $>97 \%$. PCL was purchased from Pertorp UK Limited Inc. PCL powder was dissolved in dichloromethane (Sigma Aldrich, USA), and then NDs were suspended in the PCL solution. The mixed suspension was sonicated for $15 \mathrm{~min}$. Then we injected the suspension into the manufacturing system. A tubular mold was placed horizontally under a multi-injector nozzle. The mold was rolled counterclockwise when the ND/PCL solution was sprayed on it. After the first-layer manufacturing was completed, oriented microneedles were protruded from the tubular mold, and aligned micropores were added on the first layer. The microneedles were retreated after completing punching pores. Then the sprayer started to inject the solution again and formed the second layer. After the second layer was solid, microneedles added aligned micropores on the second layer. These pores were staggered compared with those on the first layer. The manufacturing and punching of the third layer proceeded in the same manner. Finally, the tubular mold was removed.

We characterized the surface morphology of the ND/ PCL nerve bridges using scanning electron microscopy (SEM; VEGA3, Czech). We also evaluated ND size and structure using transmission electron microscopy (TEM; Talos L120C, USA). We performed gold coating for $60 \mathrm{~s}$ on the scaffold samples after we prepared ultrathin ND/ PCL conduit transverse sections. These images were taken at $\times 2000, \times 5000$, and $\times 10,000$ at an accelerating voltage of $10 \mathrm{kV}$. We evaluated randomly chosen pictures and observed the surface characterization of the materials. In addition, we measured the mechanical properties of the ND/PCL and PCL nerve bridges. We calculated the scaffold thickness and the surface elastic modulus by nanoindentation (Nano Indenter G200, Agilent, USA) and generated elastic recovery curves.

\section{Cell culture and proliferation assay}

Rat SCs (RSC96) and RAW264.7 cells were purchased from the cell bank of the Chinese Academy of Sciences (Shanghai, China). Both were cultured in high-glucose Dulbecco's modified Eagle's medium supplemented with $10 \%$ heat-inactivated fetal bovine serum (Sigma Aldrich, USA) and $1 \%$ penicillin/streptomycin solution (Sigma 
Aldrich, USA). The cells were cultured in a humidified incubator containing $5 \% \mathrm{CO}_{2}$ at $37^{\circ} \mathrm{C}$. We sterilized the ND/PCL and PCL nerve bridges using $75 \%$ ethanol (Sigma Aldrich, USA) immersion and ultraviolet light exposure. SCs were cultured at a starting concentration of $10^{4}$ cells $\mathrm{cm}^{-2}$ with $1 \% \mathrm{wt} / \mathrm{wt}, 2 \% \mathrm{wt} / \mathrm{wt}, 4 \% \mathrm{wt} / \mathrm{wt}$, and $6 \% \mathrm{wt} / \mathrm{wt}$ ND/PCL nerve bridges. The cell proliferation state was evaluated at 24, 72, 120, and $168 \mathrm{~h}$. At each time point, we transferred $100 \mu \mathrm{l}$ of solution to the 96-well plate. We used a cell counting kit 8 (CCK8; Sigma Aldrich, USA) at this step. We added $10 \mu \mathrm{l}$ of working solution to the previous solution and cultured the cells for $4 \mathrm{~h}$. Finally, all the samples were evaluated under a microplate reader at a wavelength of $450 \mathrm{~nm}$ (Thermo Fischer Scientific, USA).

\section{Scanning electron microscopy}

SCs were cultured in ND/PCL and PCL nerve bridges for 3 days. The medium was replaced every other day. The starting density was $3 \times 10^{4} \mathrm{~cm}^{-2}$. Then we washed the cells using Dulbecco's phosphate-buffered saline (Gibco, USA) three times before fixing the cells with $2.5 \%$ glutaraldehyde (Sigma Aldrich, USA) for $12 \mathrm{~h}$. After the fixation solution was replaced with $1 \%$ osmic acid (Gibco, USA), the samples were kept for $2 \mathrm{~h}$ at $4{ }^{\circ} \mathrm{C}$. We then used a graded ethanol series $(30 \%, 50 \%, 70 \%, 80 \%, 90 \%$, and 95\%) to dehydrate the cell/scaffold samples three times for $1 \mathrm{~h}$. We dried the samples in the vacuum machine for half an hour before deflation. Then we removed the samples and performed gold coating to enhance the conductivity. Finally, we observed the samples under a SEM (SU8010 Hitachi, Japan).

\section{Immunofluorescence}

SCs cultured in ND/PCL and PCL nerve bridges were washed three times after the cells were cultured for 4 days. The SCs were fixed in $4 \%$ paraformaldehyde (Gibco, USA) for $20 \mathrm{~min}$ and were immersed in $0.1 \%$ Triton X-100 (Sigma Aldrich, USA) for $10 \mathrm{~min}$. The samples were then blocked using 5\% bovine serum albumin (BSA, Gibco, USA) and incubated with a primary anti-Nestin antibody (1:500, Abcam, USA) at $4{ }^{\circ} \mathrm{C}$ for $12 \mathrm{~h}$. Then the samples were incubated with the secondary Alexa Fluor 488conjugated mouse anti-rabbit IgG (1:200, Gibco, USA) at room temperature for $2 \mathrm{~h}$. Finally, we stained the nuclei using 4,6-diamidino-2 phenylindole (DAPI) (1:500, Gibco, USA). All samples were observed using EVOS Cell Imaging Systems (Thermo Fisher Scientific, USA).

\section{Western blotting (WB), enzyme-linked immunosorbent assay (ELISA), and quantitative polymerase chain reaction (qPCR)}

For the WB assay, we lysed $10^{5}$ SCs and RAW264.7 cells in radioimmunoprecipitation assay (RIPA) lysis buffer (Thermo Fisher Scientific, USA) to collect total proteins, which were quantified by the Bradford method ${ }^{21}$. We loaded $15 \mu \mathrm{l}$ samples onto the sodium dodecyl sulfate (SDS) gel. We performed SDS polyacrylamide gel electrophoresis and then transferred the samples onto polyvinylidene fluoride membranes. We then incubated the samples at $4{ }^{\circ} \mathrm{C}$ for $12 \mathrm{~h}$ with the following primary antibodies: anti-Ki67 (1:5000, Abcam, USA), anti- $\beta$-III-tubulin (anti-Tuj1, 1:5000, Abcam, USA), anti-myelin basic protein (anti-MBP, 1:5000, Abcam, USA), anti-S100 (1:5000, Abcam, USA), anti-interleukin-6 (anti-IL-6, 1:5000, Abcam, USA), anti-tumor necrosis factor- $\alpha$ (antiTNF- $\alpha, 1: 5000$, Abcam, USA), anti-cluster of differentiation 206 (anti-CD206, 1:5000, Gibco, USA), and antiinterleukin-10 (anti-IL-10, 1:5000, Abcam, USA). The expression levels of STAT-3 and NF- $\mathrm{kB}$ were evaluated by ELISA (R\&D Systems, USA) according to the manufacturer's instructions.

We extracted total RNA from $6 \times 10^{5} \mathrm{SCs}$ and RAW264.7 cells using TRIzol reagent (Gibco, USA). In brief, we lysed SCs and RAW264.7 cells, collected the lysate, isolated RNA, and performed first-strand cDNA synthesis. The samples were processed at $65^{\circ} \mathrm{C}$ for $5 \mathrm{~min}$ in a PCR machine and centrifuged at $5000 \times g$ for $5 \mathrm{~s}$. After adding the reaction components, the samples were incubated at $25^{\circ} \mathrm{C}$ for $5 \mathrm{~min}$, at $42^{\circ} \mathrm{C}$ for $60 \mathrm{~min}$, and at $70^{\circ} \mathrm{C}$ for $5 \mathrm{~min}$. Finally, the samples were centrifuged at $5000 \times g$ for $5 \mathrm{~s}$. The reaction components included RNase inhibitor $\left(50 \mathrm{U}^{-1}{ }^{-1}\right.$, Invitrogen, USA), $5 \times$ buffer (Invitrogen, USA), deoxynucleotide mix (10 mM each) (Invitrogen, USA), dithiothreitol (Invitrogen, USA), and

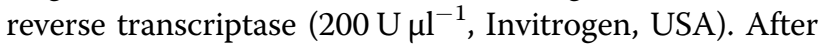
running in the PCR machine, we calculated relative quantities with the relevant program. The expression of the above genes was normalized to GAPDH. The primer sequences (forward $5^{\prime}-3^{\prime}$ ) were as follows: Ki67: ACAGGGCTTAGGAAACAGTCC; Tuj1: AGCTCACCC AGCAGATGTTC; S100: CGATGCCCCGGAAAGTTA GA; MBP: TGGGAAACCACTCTGGAAAG; TNF- $\alpha$ : CTGGGACAGTGACCTGGACT; IL-6: AGTTGCCTTC TTGGGACTGA; CD206: GGGCAATGCAAATGGAGCCG; IL-10: CATGGCCCAGAAATCAAGGA; and GAPDH: GGGAAGCCCATCACCATCTT.

\section{Animal surgery}

We selected 30 Sprague Dawley (SD) rats (male, weighing $140-150 \mathrm{~g}$ ) for in vivo assays. The rats were randomly divided into three groups: the ND/PCL nerve bridge group, the PCL nerve bridge group, and the autograft group. These rats were housed in a specific pathogen-free atmosphere. We performed peritoneal injection with $45 \mathrm{mg} \mathrm{kg}^{-1}$ pentobarbital sodium for deep anesthesia. Then we sterilized and exposed the skin, muscles, and eventually the sciatic nerve. We removed a 20-mm-long nerve segment from the greater sacrosciatic 
foramina to the popliteal space. The two nerve ends were reconnected by different nerve bridges and autografts. We dissected the sciatic nerve, reversed it by $180^{\circ}$, and used it as an autologous nerve graft. The nerve bridges were $22 \mathrm{~mm}$ in length because we sutured a 1-mm nerve stump into the lumen at each end. Then we sutured the muscles and skin. We injected penicillin intraperitoneally after surgery. Observations and procedures were carried out at 2 and 4 months, respectively. Animal care and use were authorized by the Animal Ethics Committee for Shanghai Jiao Tong University (SJTU, No. A2017072).

\section{Functional and electrophysiological tests}

We carried out walking track analysis to evaluate locomotor function. This test includes the distance between the first toe and the fifth toe (TS), the third toe and the heel (PL), and the second toe and the fourth toe (IT). We compared experimental legs (E) and normal legs (N) using the formula sciatic function index (SFI) $=$ $(-38.3 \times($ EPL - NPL $) \div$ NPL $)+(109.5 \times($ ETS - NTS $) \div$ NTS $)+(13.3 \times($ EIT - NIT $) \div$ NIT $)-8.8$. A lower SFI value indicates worse functional performance. We dissected the gastrocnemius muscle from the injured leg to calculate its weight. We also assessed sensory function apart from locomotor function. We evaluated withdrawal latency with the help of the paw withdrawal apparatus (Hargreaves Model 390, USA). Rats were placed in the testing environment for $10 \mathrm{~min}$ before heating. We exerted $25 \%$ of the maximal heat for $0.1 \mathrm{~s}$. The interval was $10 \mathrm{~min}$. Then we recorded the results with the rats standing on all four paws.

We carried out electrophysiological tests at 2 and 4 months postoperatively. Under deep anesthesia, we exposed the right sciatic nerve and fixed bipolar electrodes at two nerve stumps to deliver electrical signals. In addition, we recorded electromyography (EMG) using an electrode at the belly of the gastrocnemius muscle. We measured nerve-conducting velocity $(\mathrm{NCV})$ and distal compound motor action potential (DCMAP) using the latency and distance between nerve stumps under stimulation.

\section{Morphological tests}

We carefully cut the nerve bridges open and separated 20 -mm regenerated nerves at 2 and 4 months. We dissected the nerve samples into ultrathin 4- $\mu$ m-thick transverse sections. We assessed nerve morphological restoration using hematoxylin-eosin (HE) staining, toluidine blue (TB) staining, and TEM. In HE and TB staining, all nerve samples were fixed with $4 \%$ paraformaldehyde (Sigma Aldrich, USA) for $12 \mathrm{~h}$, and then we processed the samples using $2 \%$ osmium tetroxide and cacodylate buffer (Sigma Aldrich, USA). For TEM, we processed all nerve sections using $4 \%$ uranylacetate and lead staining working solution (Sigma Aldrich, USA) and observed the ultrathin slides under a TEM (TALOS F200X, USA) at an $80-\mathrm{kV}$ voltage. In peripheral nerve regeneration, there are some important measurements, including the number of myelinated axons, thickness of the myelin sheath, regenerated axon area, and average myelinated axon diameter. We took these measurements into account for comprehensive evaluation of the nerve structures.

We also performed HE and TB staining on the gastrocnemius muscle of the experimental sides. Random fields of view were chosen for comprehensive evaluation of the muscle fibers. We assessed the muscle using the following formula: $\mathrm{Avm}=\mathrm{Am} \div \mathrm{At} \times 100 \%$. Avm indicates the average muscle fiber area, Am represents the area of muscle fibers, and At represents the total area of the field. In addition, we performed HE staining on the major organs, such as the brain, heart, liver, spleen, lung, and kidney, to evaluate their morphological changes.

\section{Histochemical tests}

At different time points after surgery, we sacrificed the rats and prepared their regenerated nerves and muscles for immunofluorescence assays. We cut small samples of $1 \times 1 \mathrm{~cm}^{2}$ from the nerve and muscle samples. All samples were fixed in $4 \%$ paraformaldehyde (Gibco, USA) for $12 \mathrm{~h}$ at room temperature and were immersed in $1 \%$ osmium tetroxide (Sigma Aldrich, USA), dehydrated and embedded in Epon812 (Electron Microscopy Sciences, USA) resin. The samples were blocked using 5\% BSA (Gibco, USA) and incubated with primary antibodies and secondary antibodies. Finally, we stained the nuclei using DAPI (1:500, Gibco, USA). The primary antibodies used were anti-slow myosin heavy chain (1:500, Abcam, USA), anti-fast myosin heavy chain (1:500, Abcam, USA), antilaminin (1:500, Abcam, USA), anti-caspase-3 (1:500, Abcam, USA), anti-cluster of differentiation 31 (antiCD31, 1:400, Abcam, USA), anti-cluster of differentiation 34 (anti-CD34, 1:400, Abcam, USA), anti-VEGF (1:400, Abcam), anti-cluster of differentiation 68 (anti-CD68, 1:500, Abcam, USA), anti-TNF- $\alpha$ (1:500, Abcam, USA), anti-IL-6 (1:500, Abcam, USA), anti-CD206 (1:500, Abcam, USA), anti-IL-10 (1:500, Abcam, USA), anti-Tuj1 (1:300, Abcam, USA), anti-neurofilament 200 (NF200, 1:300, Abcam, USA), anti-S100 (1:300, Abcam, USA), and anti-MBP (1:300, Abcam, USA). We also prepared major organs, such as the brain, heart, liver, spleen, lung, and kidney, for histochemical assays at 4 months after surgery. We cut the middle parts transversely to prepare samples for histochemical evaluation. The primary antibodies used were anti-glial fibrillary acidic protein (anti-GFAP, 1:300, Abcam, USA) and anti-caspase-3 (1:500, Abcam, USA). The slides were observed under immunofluorescence microscopes and light microscopes (Leica, Germany). 


\section{Statistical analysis}

All tests were repeated three times, and the results are displayed as the mean \pm standard deviation. A $p$ value of 0.05 was considered significant by one-way analysis of variance and Student's $t$ test.

\section{Results}

\section{Fabrication and characterization of ND nerve bridges}

In this study, we used concentric manufacturing with multilayered spraying and gradient dotting to fabricate microporous ND/PCL nerve bridges (Fig. 1). A tubular mold was placed horizontally with a roller rotating counterclockwise. The multi-injector nozzle sprayed the ND/PCL mixed suspension vertically toward the tubular mold. After the first layer was solid, the nozzle sprayed the second ND/PCL layer onto the first layer. Unlike the conventional design of overlapped pores, we added staggered micropores to the second layer. This approach could better prevent fibroblasts from growing inside and had no negative impacts on nutrient exchange. Similarly, the third layer, which was also the outermost layer, was sprayed on the previous two layers. The concentric triplelayered structure provided appropriate elasticity and receptivity. In addition, we improved our manufacturing process using micropores smaller than those used in previous reports $^{22}$ and enabled free entrance of water and oxygen and mechanical barriers for alien cell intrusion.
We characterized the morphology of the ND/PCL nerve bridges using optical imaging and SEM. The material surface was relatively rough. The multilayered architecture in the transverse section and staggered net-shaped microporous dotting were displayed clearly at $\times 2000$, $\times 5000$ and $\times 10,000$ magnification. In addition, we evaluated ND size and structure using TEM. The diamond nanoparticles were approximately $30 \mathrm{~nm}$ in diameter distributed in the nerve bridge (Fig. 2). The ideal elastic property of the nerve bridge is important because scaffolds that are either too soft or too hard cannot promote functional long-term peripheral nerve regeneration. The elastic modulus of the ND/PCL nerve bridge was $72.88 \mathrm{MPa}$, in comparison with $35.10 \mathrm{MPa}$ for its PCL counterpart. In addition, we evaluated the elongation at break of both materials. This value was $48.65 \%$ in the ND/ PCL nerve bridge versus $36.98 \%$ in its PCL counterpart. The excellent mechanical characteristics ensured longterm in vivo structural support for neurite sprouting (Fig. 2).

\section{Cell proliferation in ND nerve bridges}

To evaluate different ND concentrations in the PCL substrate, we performed a CCK8 assay using 1\%, 2\%, $4 \%$ and $6 \% \mathrm{ND} / \mathrm{PCL}$ nerve bridges. At 24, 72, 120, and $168 \mathrm{~h}$, we evaluated cell proliferation in the four groups and found that the $2 \% \mathrm{ND} / \mathrm{PCL}$ nerve bridge group was more suitable for SC proliferation than the remaining groups

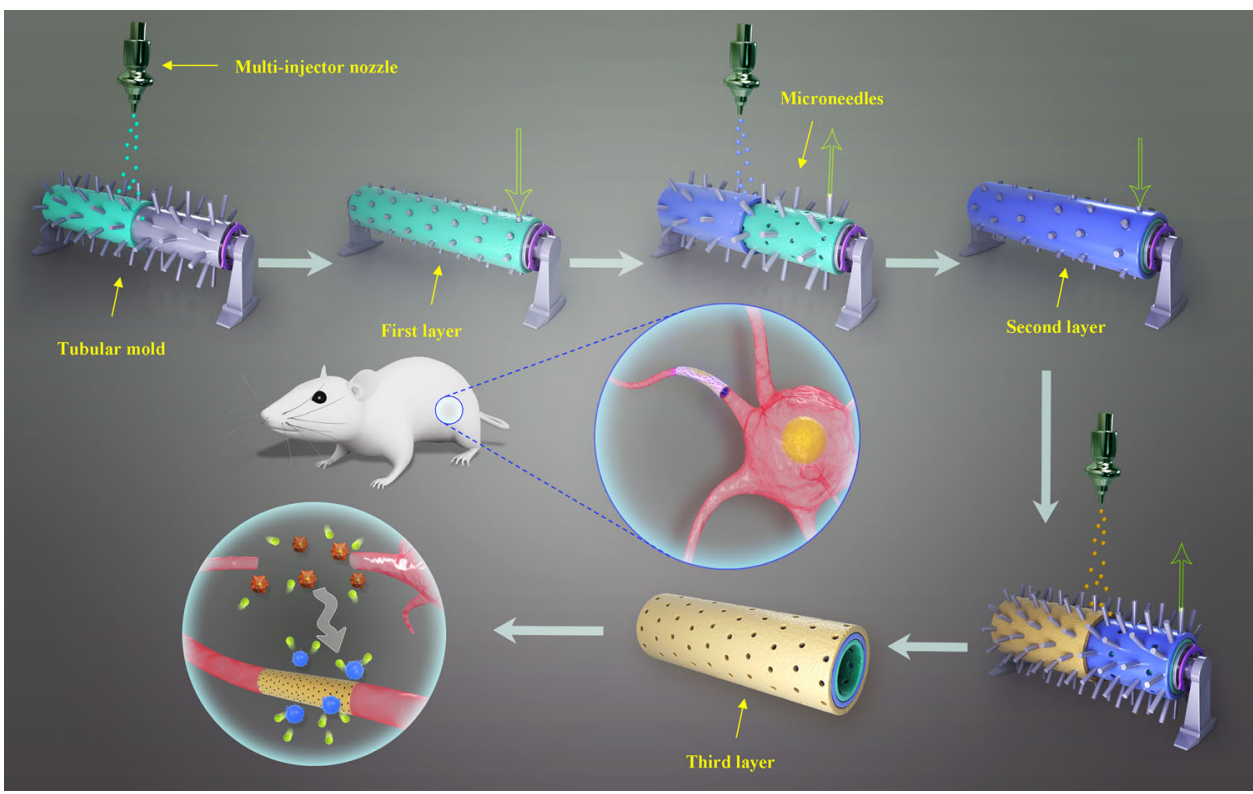

Fig. 1 Schematic introduction of the nanodiamond/polycaprolactone (ND/PCL) nerve bridge manufacturing process and in vivo performance. A tubular mold was placed horizontally with a roller rotating counterclockwise. The multi-injector nozzle sprayed the ND/PCL mixed solution vertically toward the tubular mold. Micropores were created on the ND/PCL layer. After the first layer was solid, the nozzle sprayed the second ND/PCL layer onto the first layer. Staggered micropores were added to the second layer. Similarly, the third layer, which was also the outermost layer, was sprayed on the previous two layers. The concentric triple-layered structure provided appropriate elasticity and receptivity. In addition, this structure regenerated peripheral nerves and induced macrophage polarization in vivo. The yellow arrow refers to the components 

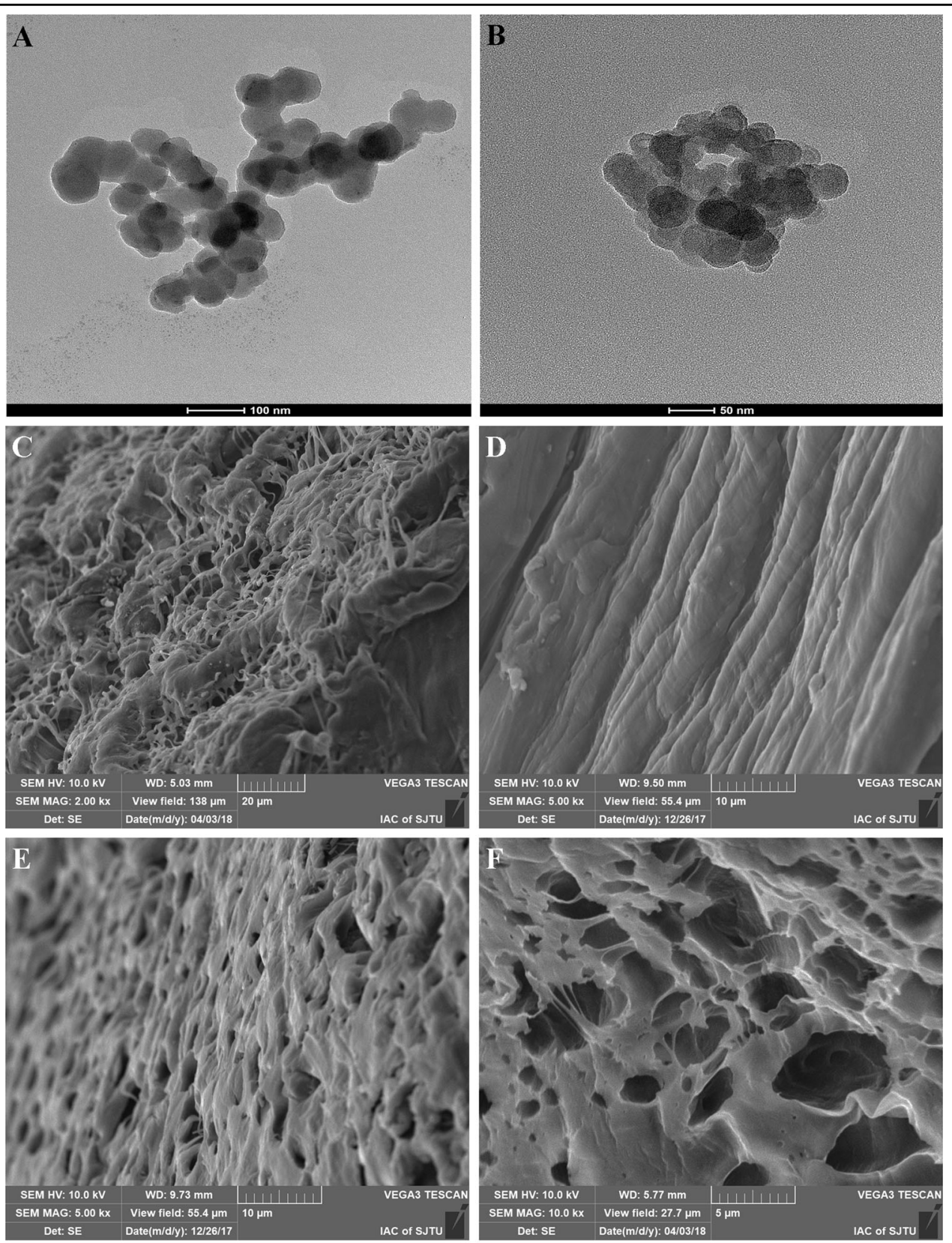

G

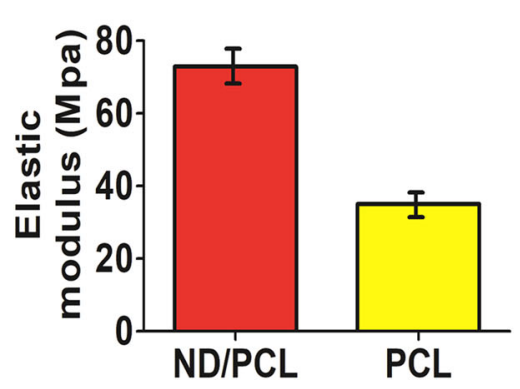

$\mathbf{H}$

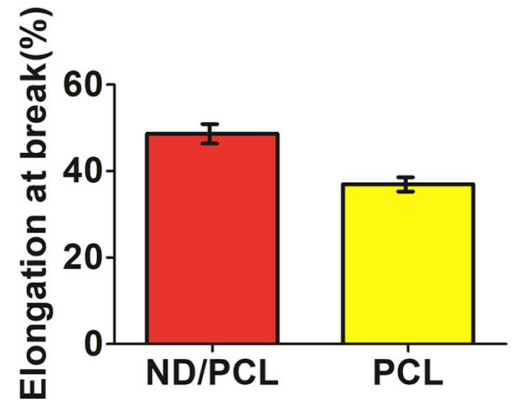

Fig. 2 Characterization of diamond nanoparticles and the nanodiamond/polycaprolactone (ND/PCL scaffold. Morphology of diamond nanoparticles by transmission electron microscopy, scale bar $=100 \mathrm{~nm}$ (a) and $50 \mathrm{~nm}$ (b). Surface morphology of the ND/PCL nerve bridges by scanning electron microscopy. Rough surface layer (c) and multilayered structure (d) of the ND/PCL nerve bridges. Staggered micropores in the scaffold surface (e, f). Scale bars: $20 \mu \mathrm{m}$ (c), $10 \mu \mathrm{m}$ (d), $10 \mu \mathrm{m}$ (e), $5 \mu \mathrm{m}$ (f). Mechanical properties' evaluation of ND/PCL and PCL nerve bridges. Elastic modulus (Mpa) (g). Elongation at break (\%) (h) 
(Fig. S1). We decided to use 2\% ND/PCL in the following experiments because the SCs in the $4 \%$ and $6 \% \mathrm{ND} / \mathrm{PCL}$ nerve bridge groups were significantly less proliferative than those in the $2 \%$ group. In addition to the CCK assay, we also performed WB and qPCR and evaluated cell proliferation using Ki67. The Ki67 expression level was significantly higher with the ND/PCL nerve bridges than with their PCL counterparts by 3.5-fold $(p<0.05)$ (Fig. 3). These results showed the potential of the ND nerve bridges to support SC proliferation.

\section{Cell morphology in ND nerve bridges}

To evaluate cell morphology and viability on the scaffolds, we performed SEM after 3 days of cell culture in ND nerve bridges. Cells were distributed widely in the scaffolds and covered most of the fields. We observed better extension of SC protuberances in the ND/PCL nerve bridge than in its PCL counterpart (Fig. 3). The results indicated ideal cell viability and attachment to the ND nerve bridge.

\section{Cell neural expression in ND nerve bridges}

To evaluate SC neural properties in ND nerve bridges, we included some neural markers, such as S100, Tuj1, MBP, and Nestin, and we performed WB and qPCR to investigate their expression. The S100 expression level was 4.3 -fold higher with the ND/PCL nerve bridges than with their PCL counterparts $(p<0.05)$. The Tuj1 expression level was 8.3-fold higher with the ND/PCL nerve bridges than with their PCL counterparts $(p<0.05)$. The MBP expression level was 2.7-fold higher with the ND/ PCL nerve bridges than with their PCL counterparts $(p<$ 0.05 , Fig. 3). We also performed an immunofluorescence assay to evaluate Nestin expression levels in both scaffolds. The green fluorescence intensity was also higher in the ND/PCL nerve bridge than in its PCL counterpart (Fig. S2). These results revealed the excellent neural maintenance of the ND nerve bridges.

\section{Macrophage polarization in ND nerve bridges}

To evaluate the ability of the ND nerve bridges to regulate the macrophage phenotype, we performed WB and qPCR and investigated IL-6, TNF- $\alpha$, CD206, and IL-10 expression levels after we cultured RAW264.7 cells in ND/PCL nerve bridges and their PCL counterparts for $72 \mathrm{~h}$. The results showed that macrophages in the ND/ PCL nerve bridges showed significantly lower expression levels of IL- 6 and TNF- $\alpha$ and higher expression levels of CD206 and IL-10 than their PCL counterparts (Fig. 3). The IL-6 expression level was 6.2-fold lower in the ND/ PCL nerve bridges than in their PCL counterparts $(p<$ $0.05)$. The TNF- $\alpha$ expression level was 2.1 -fold lower in the ND/PCL nerve bridges than in their PCL counterparts $(p<0.05)$. The CD206 expression level was 2.4 -fold higher in the ND/PCL nerve bridges than in their PCL counterparts $(p<0.05)$. The IL-10 expression level was 2.0 -fold higher in the ND/PCL nerve bridges than in their PCL counterparts $(p<0.05$, Fig. 3$)$. In addition, we evaluated STAT -3 and NF- $\mathrm{KB}$ release and found that, compared with the PCL scaffold, the ND/PCL nerve bridges significantly upregulated STAT-3 and downregulated NF- $\kappa$ B expression levels in RAW264.7 cells $(p<0.05$, Fig. 3). These results initially proved that the ND nerve bridges could induce macrophage polarization into the M2 type in vitro.

\section{Functional recovery of the sciatic nerve in ND nerve bridges}

In vivo investigation further helps us understand the glioprotective and regenerative roles of ND/PCL nerve bridges. We created a $20-\mathrm{mm}$ sciatic nerve defect in the SD rat model. The rats were randomly allocated into three groups: the ND/PCL nerve bridge group, the PCL nerve bridge group, and the nerve autograft group. We evaluated sciatic nerve recovery at 2 and 4 months after surgery. The conduit and nerve were recorded using optical imaging (Fig. S3). We did not find severe complications, such as delayed wound healing, skin ulcers, or superficial infection, within the first 2 weeks after surgery.

We performed walking track analysis to assess the locomotor performance using SFI. The SFI value was significantly higher with autografts than with ND/PCL and PCL nerve bridges at 2 months postoperatively $(p<$ 0.05 , Fig. S4). However, this parameter was similar between the autograft and ND/PCL nerve bridge groups at 4 months postoperatively $(p>0.05)$. The PCL nerve bridge group displayed a much poorer SFI value than the other groups $(p<0.05)$. In addition to locomotor performance, we also investigated sensory recovery by examining withdrawal latency. The reaction duration was significantly shortened in the ND/PCL and autograft groups compared with that in the PCL group at 2 and 4 months after surgery $(p<0.05$, Fig. S4). These results showed that the ND/PCL nerve bridges improved locomotor and sensory recovery after severe peripheral nerve injury.

\section{Electrophysiological recovery of the sciatic nerve in ND nerve bridges}

We evaluated sciatic nerve bioelectrical activity with two indices, NCV and DCMAP, by EMG. At 2 months after surgery, NCV was significantly higher in autografts $\left(27.6 \mathrm{~m} \mathrm{~s}^{-1}\right)$ than in ND/PCL and PCL nerve bridges $\left(16.4 \mathrm{~m} \mathrm{~s}^{-1}, 9.5 \mathrm{~m} \mathrm{~s}^{-1}\right)(p<0.05)$. Nevertheless, this parameter showed an insignificant difference between ND/ PCL nerve bridges $\left(27.2 \mathrm{~m} \mathrm{~s}^{-1}\right)$ and autografts $\left(31.9 \mathrm{~m} \mathrm{~s}^{-1}\right)$ at 4 months after surgery. The NCV of the PCL nerve bridges was $16.3 \mathrm{~m} \mathrm{~s}^{-1}$, much lower than that of the other 


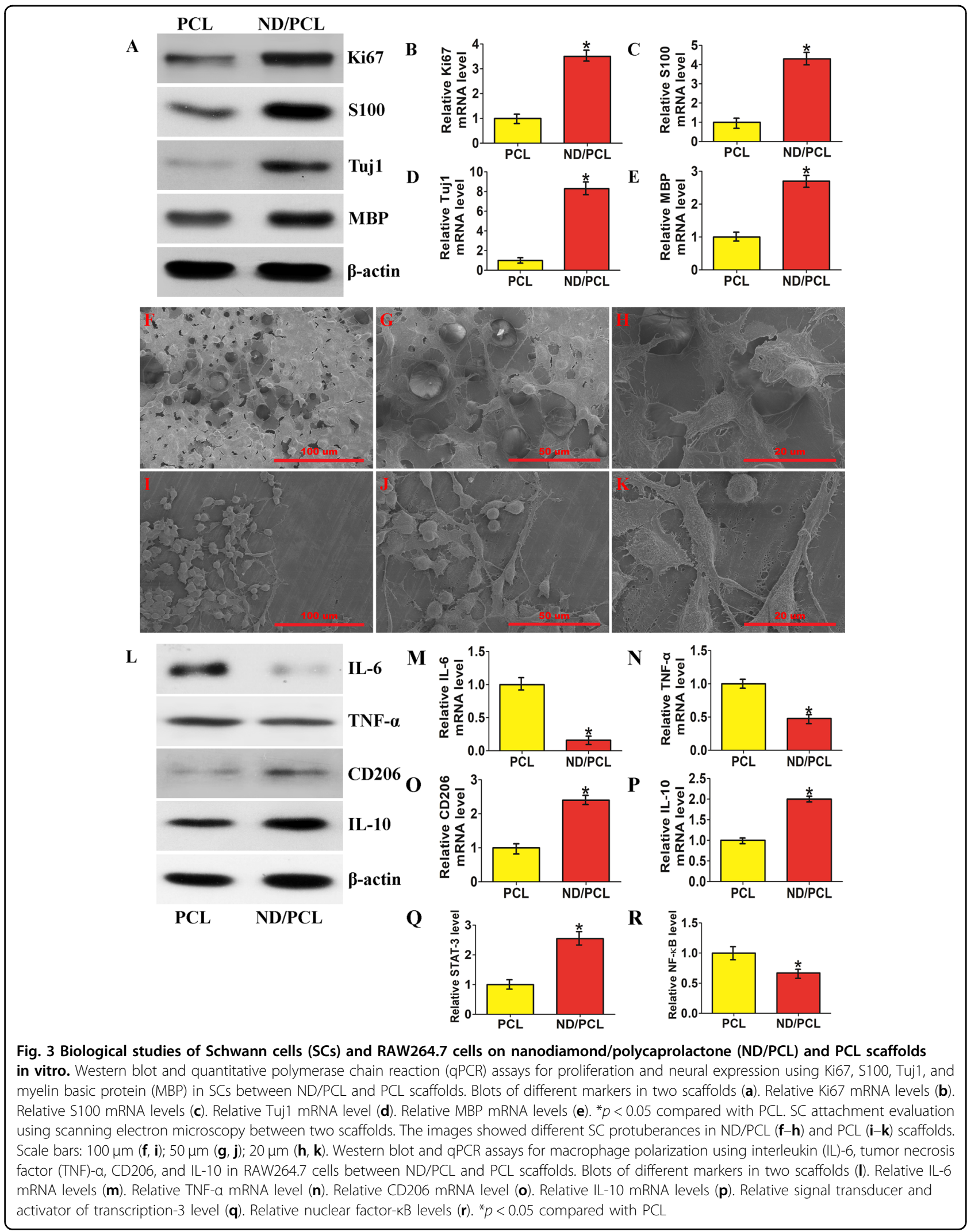


groups $(p<0.05$, Fig. S4). DCMAP is the total action potential of every motor endplate and is an important indicator of muscle contraction gain. The DCMAP of the autograft groups $(21.9 \mathrm{mV})$ outnumbered that of ND/PCL $(14.1 \mathrm{mV})$ and PCL $(7.6 \mathrm{mV})$ nerve bridges at 2 months after surgery $(p<0.05)$. However, the DCMAP value of the ND/PCL nerve bridges $(29.7 \mathrm{mV})$ was similar to that of the autografts $(25.9 \mathrm{mV})$ at 4 months after surgery $(p>$ $0.05)$ and significantly higher than that of their PCL counterparts $(18.3 \mathrm{mV})(p<0.05$, Fig. S4). These results showed the improved bioelectrical activity of the regenerated sciatic nerves in the ND/PCL nerve bridges.

\section{Morphological regeneration of the sciatic nerve in ND nerve bridges}

We performed HE staining, TB staining, and TEM using 20-mm regenerated sciatic nerve samples separated from nerve bridges or the autografts themselves. We evaluated several indicators for sciatic nerve repair, including myelinated axon number, myelin sheath thickness, regenerated axon area, and average myelinated axon diameter. The regenerated axon area was significantly greater in the autograft group than in the other groups at 2 and 4 months after surgery. However, the number of myelinated axons, thickness of the myelin sheath, and average myelinated axon diameter values were similar between autografts and ND/PCL nerve bridges $(p>0.05)$. Both groups showed significantly higher values than their PCL counterparts at 2 and 4 months $(p<0.05$, Fig. 4$)$. We investigated the thickness and average diameter of myelinated fibers using TEM observation. The results were similar to the previous morphological staining results. Higher values for the thickness and average diameter of myelinated fibers were achieved in autografts and ND/ PCL nerve bridges than in their PCL counterparts. These results confirmed that the ND/PCL nerve bridges could promote sciatic nerve morphological repair.

\section{Muscle viability and strength improved by ND nerve bridges}

We evaluated the gastrocnemius muscle weight and observed muscle morphological changes as well as muscle fiber types (fast and slow types). At 2 months after surgery, the gastrocnemius muscle weight was evidently higher with the ND/PCL nerve bridges than with the PCL nerve bridges $(p<0.05)$. However, this weight was highest in the autograft group. At 4 months after surgery, the results did not reveal any difference between the ND/PCL nerve bridges and autografts $(p>0.05)$. In addition, we performed $\mathrm{HE}$ and TB staining on the muscle samples. We noticed that the muscle fiber was oriented and aligned in the ND/PCL nerve bridge and autograft groups. Fast twitch phenotype fibers filled the major regions. The muscle samples in the PCL nerve bridge group were irregular and incomplete in comparison. Slow twitch-type muscle fibers occupied most regions. We also performed laminin and caspase immunofluorescence staining on the muscle samples from the three groups. A higher laminin expression level and less apoptosis were observed with autografts and ND/PCL nerve bridges than with their PCL counterparts $(p<0.05$, Fig. 5$)$. These results showed that the ND/PCL nerve bridges could significantly enhance skeletal muscle regeneration, reduce muscle apoptosis, and alleviate muscle atrophy.

\section{Angiogenesis in sciatic nerve repair stimulated by ND nerve bridges}

To evaluate the angiogenesis state in the regenerated nerves, we performed CD31, CD34, and VEGF immunofluorescence staining. Angiogenesis was assessed using several important factors, such as microvessel density, vessel-like structure (VLS) area, and density ((VLS area + CD31 $\div$ CD34 + area $\div$ total scaffold area). CD31, CD34, and VEGF expression levels were significantly higher in ND/PCL nerve bridges than in PCL nerve bridges at 2 and 4 months after surgery $(p<0.05)$. However, the values of both groups were much lower than those of the autograft group $(p<0.05$, Fig. 6 ). These results indicated that the ND/PCL nerve bridges stimulated angiogenesis to a limited extent in long-term peripheral nerve regeneration.

\section{Neural activity restoration in ND nerve bridges}

The sciatic nerves express certain neural proteins, such as S100, Tuj1, MBP, and NF200. We evaluated these markers in the three experimental groups. S100 and MBP expression levels were slightly higher in the ND/PCL and autograft groups than in their PCL counterparts $(p>0.05)$. However, Tuj1 and NF200 expression showed a similar fluorescence intensity between the ND/PCL nerve bridges and autografts, both of which were significantly higher than their PCL counterpart $(p<0.05$, Figs. 7 and 8$)$. In addition to neural evaluation of sciatic nerves, we also performed GFAP immunofluorescence staining on brain tissues from all groups. The results showed that the GFAP expression level was significantly higher in the ND/PCL nerve bridge and autograft groups than in their PCL counterparts $(p<0.05$, Fig. 9). This was consistent with the sciatic nerve, indicating that the central nervous system might be regulated and influenced by peripheral nerve insult. These results indicated that the ND nerve bridges could restore the neural expression of peripheral nerves to a certain extent.

\section{In vivo macrophage polarization mediated by ND nerve bridges}

We evaluated macrophage expression and confirmed the different phenotypes of regenerated sciatic nerves at 3 days, 14 days, and 4 months after nerve injury. We identified macrophages in the regenerated nerve tissues 
A

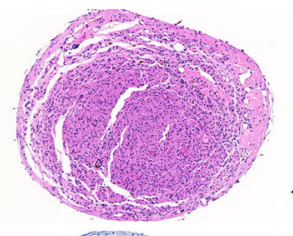

D
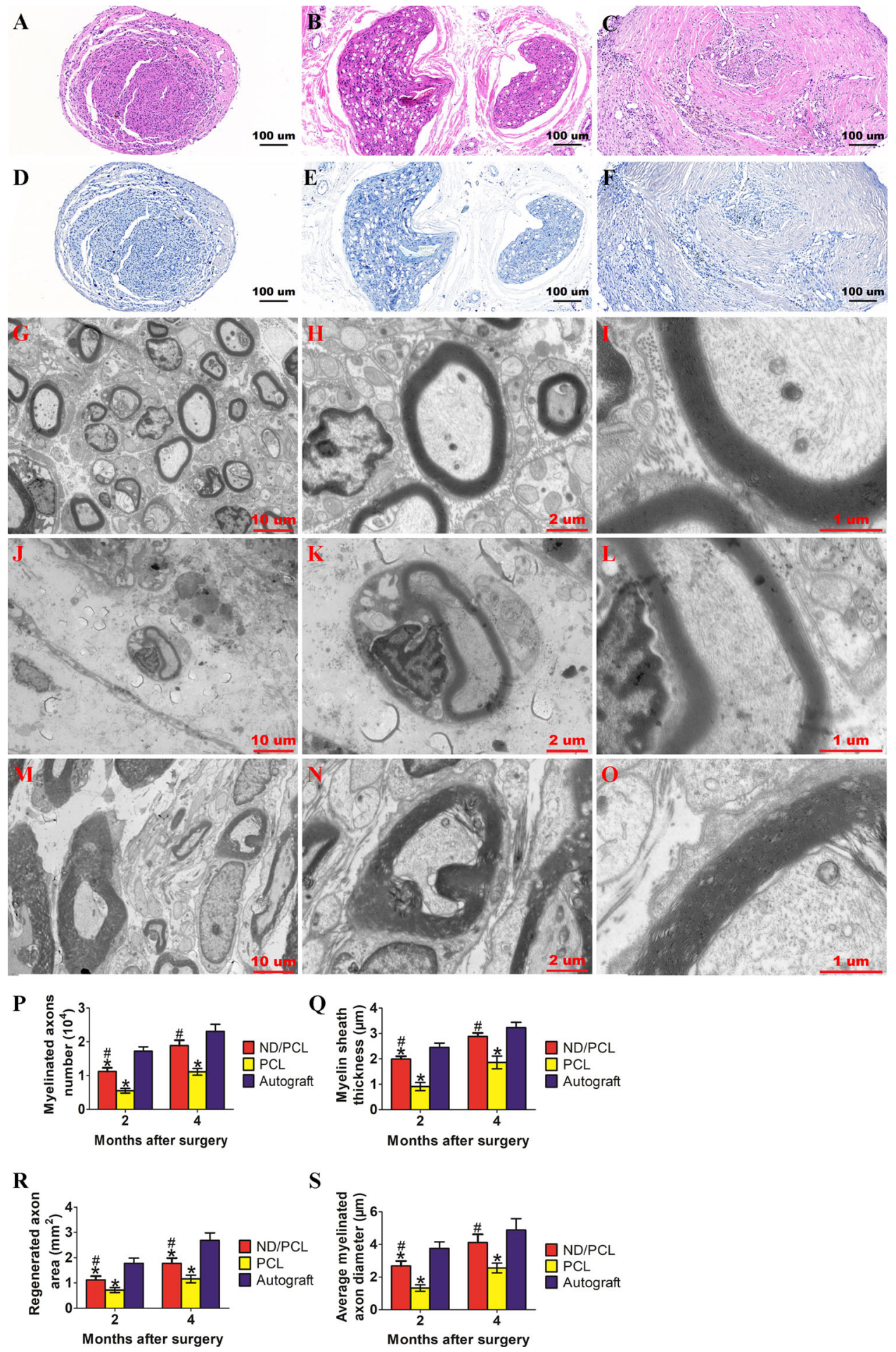

S

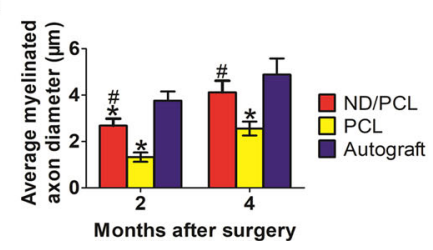

Fig. 4 Morphological recovery of sciatic nerves from the nanodiamond/polycaprolactone (ND/PCL), PCL, and autograft groups.

Regenerated sciatic nerve morphology using hematoxylin-eosin $(\mathbf{a}-\mathbf{c})$ and toluidine blue $(\mathbf{d}-\mathbf{f})$ staining at 4 months postoperatively. ND/PCL nerve bridge $(\mathbf{a}, \mathbf{d})$. PCL nerve bridge (b, e). Autograft $(\mathbf{c}, \mathbf{f})$. Scale bar $=100 \mu \mathrm{m}$. Axon regrowth and remyelination evaluation of regenerated sciatic nerves using transmission electron microscopy at 4 months postoperatively. ND/PCL nerve bridge $(\mathbf{g}-\mathbf{i})$. PCL nerve bridge $(\mathbf{j}-\mathbf{I})$. Autograft $(\mathbf{m}-\mathbf{o})$. Scale bars: $10 \mu \mathrm{m}(\mathbf{g}, \mathbf{j}, \mathbf{m}) ; 2 \mu \mathrm{m}(\mathbf{h}, \mathbf{k}, \mathbf{n}) ; 1 \mu \mathrm{m}(\mathbf{i}, \mathbf{l}, \mathbf{o})$. Myelinated axon number $\left(10^{4}\right)(\mathbf{p})$. Myelin sheath thickness $(\mu \mathrm{m})(\mathbf{q})$. Regenerated axon area $\left(\mathrm{mm}^{2}\right)(\mathbf{r})$. Average myelinated axon diameter $(\mu \mathrm{m})(\mathbf{s}) .{ }^{*} p<0.05$ compared with autograft, ${ }^{\#} p<0.05$ compared with $\mathrm{PCL}$ 

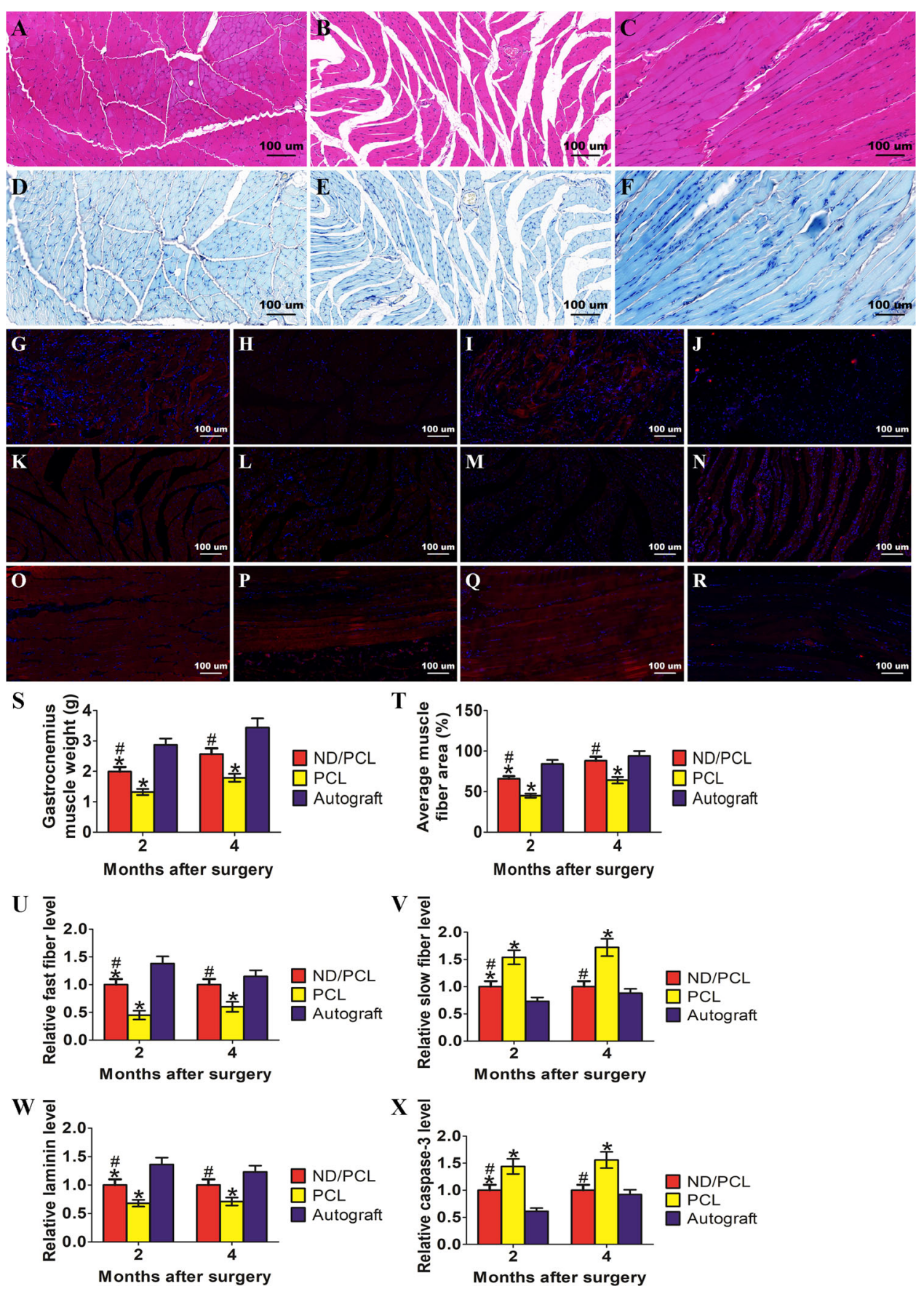

Fig. 5 Muscle recovery in the nanodiamond/polycaprolactone (ND/PCL), PCL, and autograft groups. Muscle morphology using hematoxylin-eosin $(\mathbf{a}-\mathbf{c})$ and toluidine blue $(\mathbf{d}-\mathbf{f})$ staining at 4 months postoperatively. ND/PCL nerve bridge $(\mathbf{a}, \mathbf{d})$. PCL nerve bridge $(\mathbf{b}, \mathbf{e})$. Autograft (c, $\mathbf{f})$. Scale bar $=100 \mu \mathrm{m}$. Muscle viability evaluation using fast-type myosin $(\mathbf{g}, \mathbf{k}, \mathbf{o})$, slow-type myosin $(\mathbf{h}, \mathbf{l}, \mathbf{p})$, laminin $(\mathbf{i}, \mathbf{m}, \mathbf{q})$, and caspase-3 (j, $\mathbf{n}, \mathbf{r})$ at 4 months postoperatively. ND/PCL nerve bridge $(\mathbf{g}-\mathbf{j})$. PCL nerve bridge $(\mathbf{k}-\mathbf{n})$. Autograft $(\mathbf{o}-\mathbf{r})$. Scale bar $=100 \mu$ m. Muscle viability evaluation at 2 and 4 months postoperatively. Gastrocnemius muscle weight $(\mathrm{g})(\mathbf{s})$. Average muscle fiber area (\%) (t). Relative fast fiber level (u). Relative slow fiber level $(\mathbf{v})$. Relative laminin level $(\mathbf{w})$. Relative caspase-3 levels $(\mathbf{x}) .{ }^{*} p<0.05$ compared with autograft, ${ }^{*} p<0.05$ compared with $\mathrm{PCL}$

using the surface marker CD68. The expression levels of the M1 phenotype markers IL-6 and TNF- $\alpha$ were significantly lower in the ND/PCL nerve bridges than in their PCL counterparts at different time points $(p<0.05)$. The autograft samples showed the lowest IL- 6 and TNF- $\alpha$ expression levels among all groups. The expression levels of the M2 phenotype markers CD206 and IL-10 were similar in the ND/PCL nerve bridge and autograft groups 


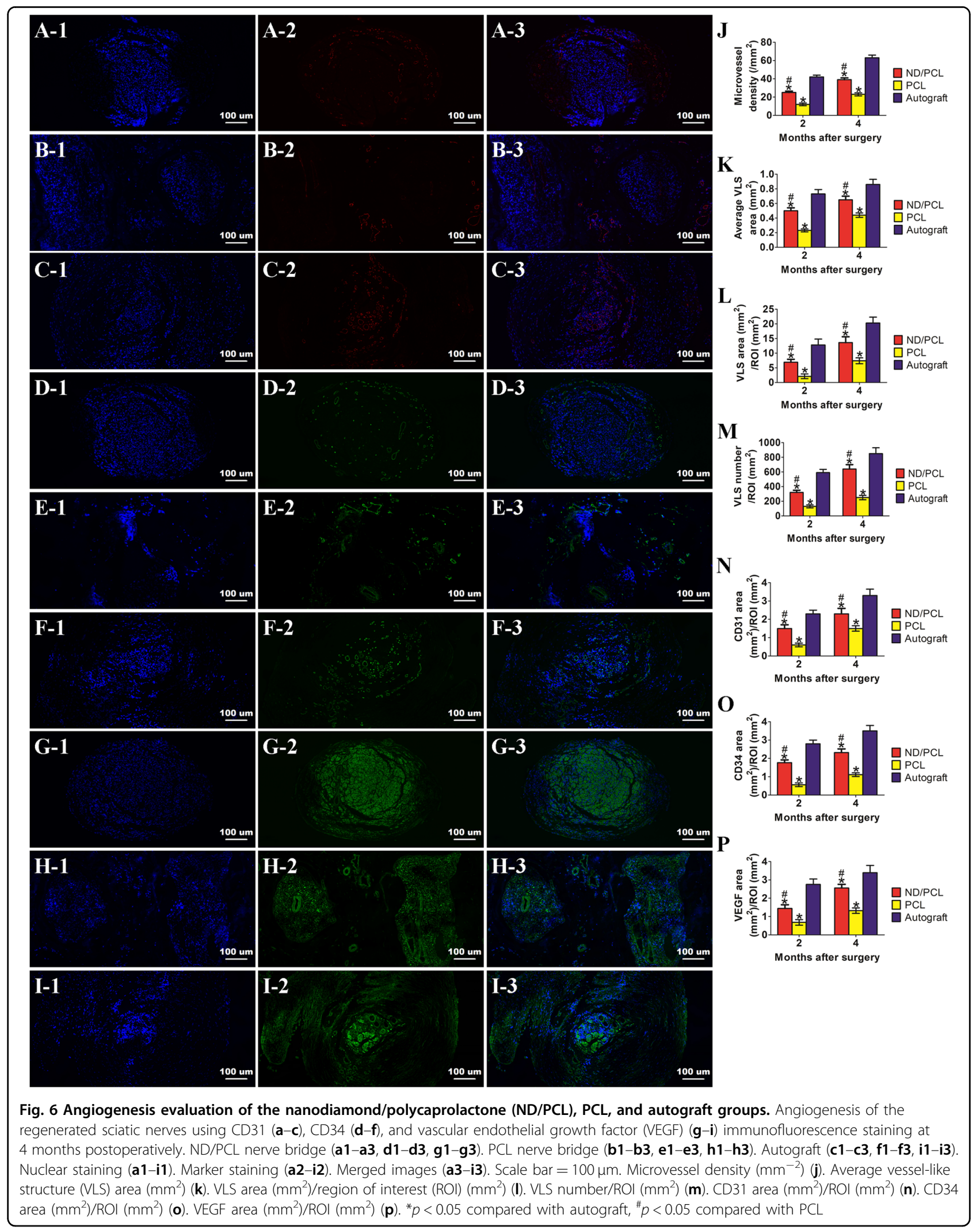




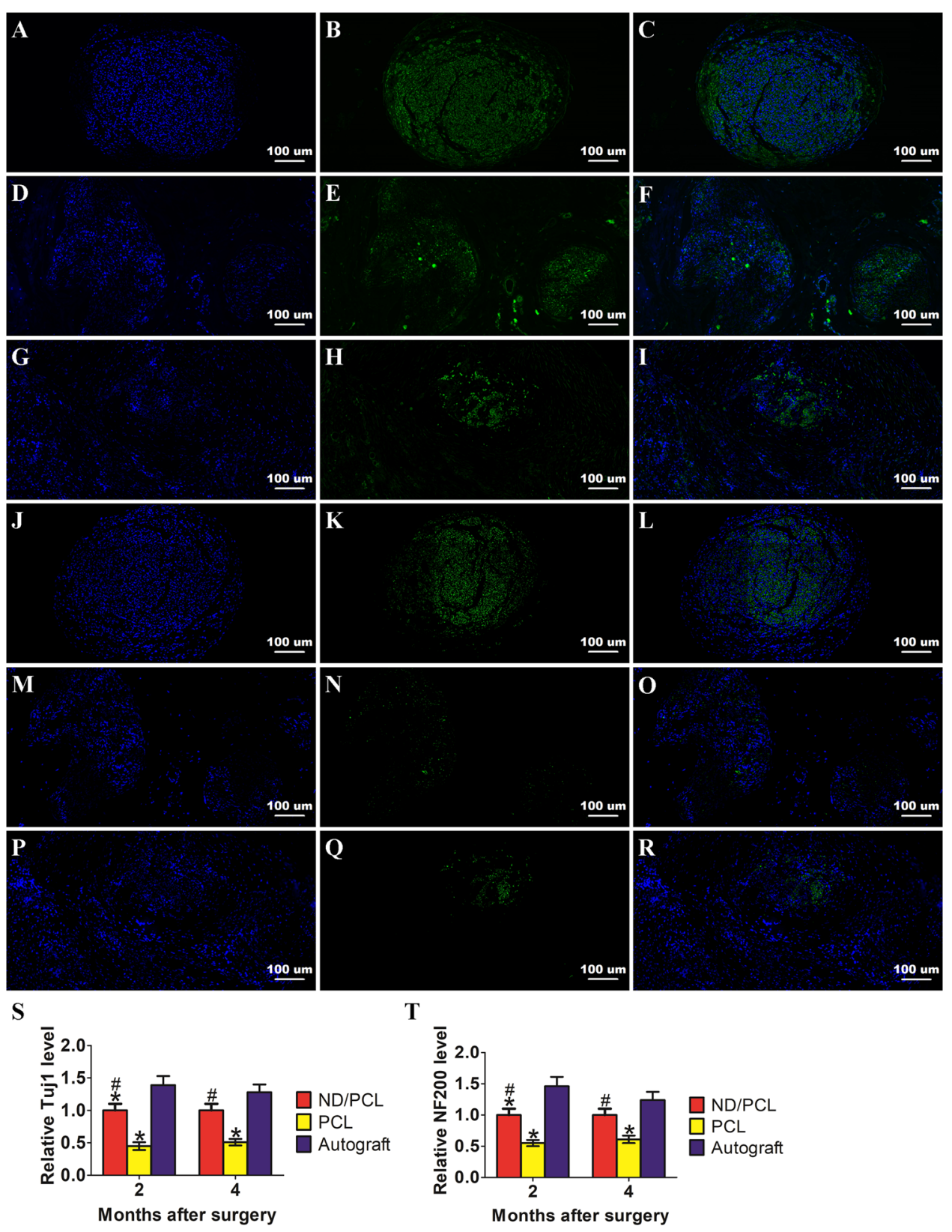

Fig. 7 Immunofluorescence assay of regenerated sciatic nerves using Tuj1 and NF200 for axon regeneration assessment at 4 months postoperatively. Immunofluorescence assay of regenerated sciatic nerves using Tuj1 (a-i) and NF200 (j-r) for axon regeneration assessment at 4 months postoperatively. Nanodiamond/polycaprolactone (PCL) nerve bridge $(\mathbf{a}-\mathbf{c}, \mathbf{j}-\mathbf{I})$. PCL nerve bridge $(\mathbf{d}-\mathbf{f}, \mathbf{m}-\mathbf{0})$. Autograft $(\mathbf{g}-\mathbf{i}, \mathbf{p}-\mathbf{r})$. Nuclear staining (a, d, $\mathbf{g}, \mathbf{j}, \mathbf{m}, \mathbf{p})$. Marker staining ( $\mathbf{b}, \mathbf{e}, \mathbf{h}, \mathbf{k}, \mathbf{n}, \mathbf{q})$. Merged images $(\mathbf{c}, \mathbf{f}, \mathbf{i}, \mathbf{I}, \mathbf{o}, \mathbf{r})$. Scale bar $=100 \mu \mathrm{m}$. Relative Tuj1 level (s). Relative NF200 level (t). ${ }^{*} p<0.05$ compared with autograft, ${ }^{*} p<0.05$ compared with $\mathrm{PCL}$

and were significantly higher than those in their PCL counterparts at 3 and 14 days $(p<0.05$, Figs. 10-14). Nevertheless, no differences in CD206 or IL-10 expression levels were displayed among the three groups at 4 months $(p>0.05$, Figs. 10-14). These results revealed that the ND/PCL nerve bridges could induce M1 phenotype macrophages into the M2 phenotype and ameliorate the immune milieu for better peripheral nerve regeneration.

\section{Long-term in vivo biosafety investigation of ND nerve bridges}

We performed pathological examination of major functioning organs, such as the brain, heart, liver, spleen, lung, and kidney, 4 months after nerve injury. We did not observe evident morphological changes in the ND/PCL nerve bridge, PCL nerve bridge, or autograft groups $(p>$ 0.05, Fig. 15). In addition, we evaluated tissue apoptotic 


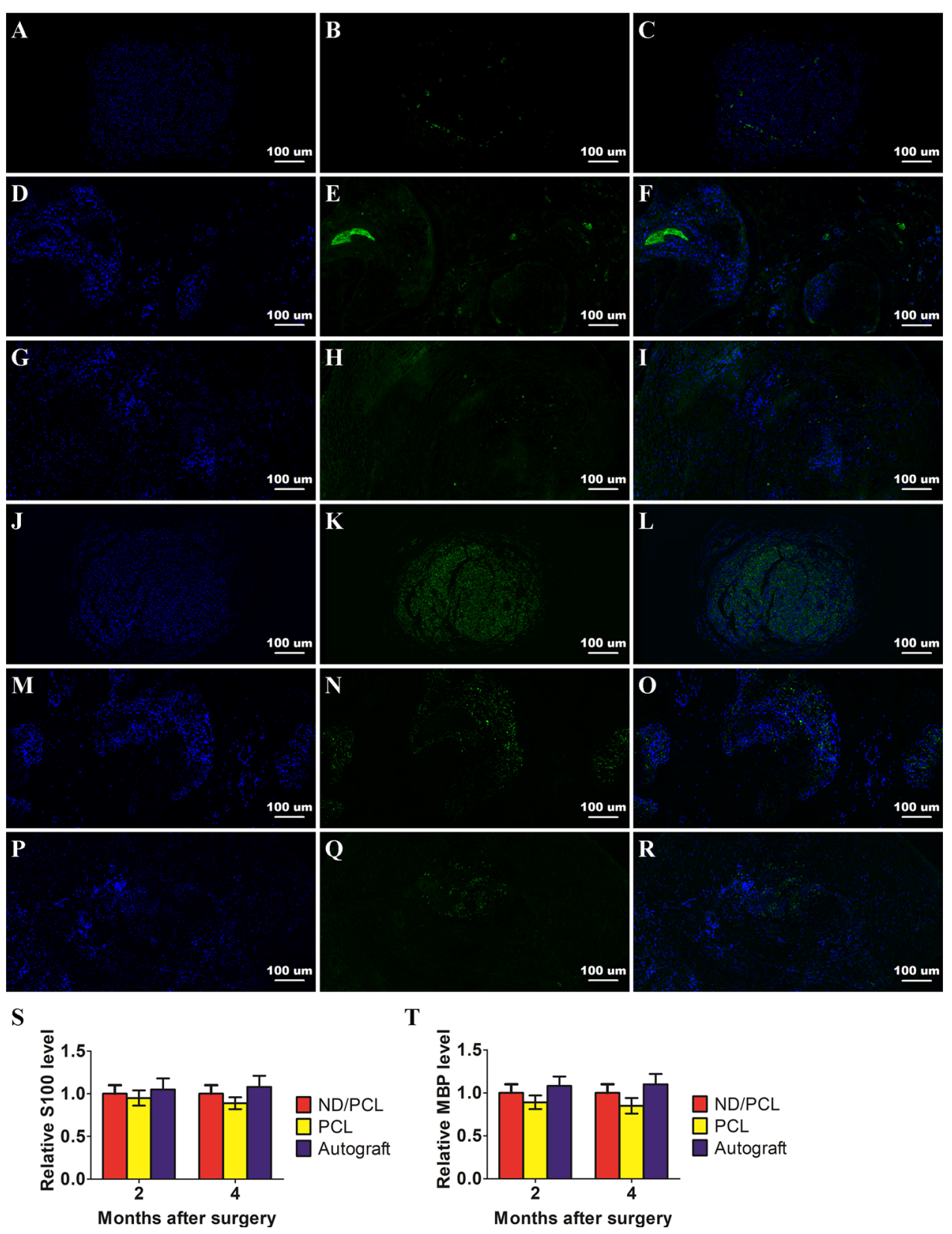

Fig. 8 Immunofluorescence assay of regenerated sciatic nerves using S100 and myelin basic protein (MBP) for nerve regeneration evaluation at $\mathbf{4}$ months postoperatively. Immunofluorescence assay of regenerated sciatic nerves using S100 (a-i) and MBP $(\mathbf{j}-\mathbf{r})$ for nerve regeneration evaluation at 4 months postoperatively. Nanodiamond/polycaprolactone $(P C L)$ nerve bridge $(\mathbf{a}-\mathbf{c}, \mathbf{j}-\mathbf{l})$. PCL nerve bridge $(\mathbf{d}-\mathbf{f}, \mathbf{m}-\mathbf{o})$. Autograft ( $\mathbf{g}-\mathbf{i}, \mathbf{p}-\mathbf{r})$. Nuclear staining $(\mathbf{a}, \mathbf{d}, \mathbf{g}, \mathbf{j}, \mathbf{m}, \mathbf{p})$. Marker staining $(\mathbf{b}, \mathbf{e}, \mathbf{h}, \mathbf{k}, \mathbf{n}, \mathbf{q})$. Merged images $(\mathbf{c}, \mathbf{f}, \mathbf{i}, \mathbf{I}, \mathbf{o}, \mathbf{r})$. Scale bar $=100 \mu \mathrm{m}$. Relative S100 level (s). Relative MBP level (t)

conditions by performing a caspase-3 histochemical assay. The results showed that the caspase- 3 expression level of the liver was higher in the PCL nerve bridges than in their $\mathrm{ND} / \mathrm{PCL}$ counterparts $(p<0.05)$, which was close to the autograft. This parameter did not show any significant difference in other organs $(p>0.05$, Figs. S5 and S6). These results validated that the ND/PCL nerve bridges had excellent biocompatibility in the living body without inducing any toxic effects and contributed to tissue proliferation in the long term.

\section{Discussion}

Advanced biomaterials are playing an increasingly important role in nerve tissue regeneration ${ }^{23}$. For severe long-range peripheral nerve defects, autografts cannot meet the present demands and may have some negative 


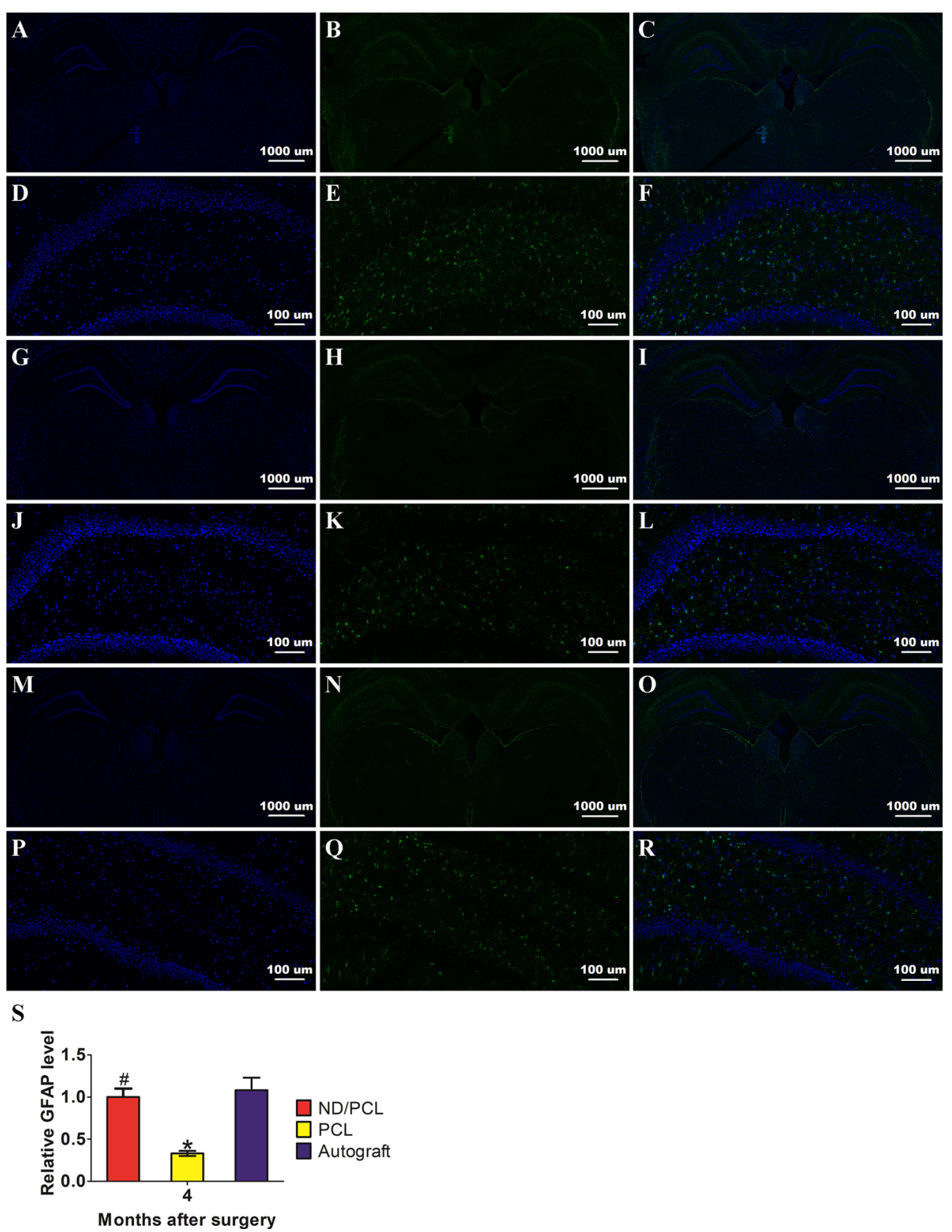

Fig. 9 Immunofluorescence assay of brains using glial fibrillary acidic protein (GFAP) for neural expression evaluation at 4 months postoperatively. Nanodiamond/polycaprolactone (PCL) nerve bridge (a-f). PCL nerve bridge $(\mathbf{g}-\mathbf{l})$. Autograft $(\mathbf{m}-\mathbf{r})$. Nuclear staining $(\mathbf{a}, \mathbf{d}, \mathbf{g}, \mathbf{j}, \mathbf{m}, \mathbf{p})$. GFAP staining $(\mathbf{b}, \mathbf{e}, \mathbf{h}, \mathbf{k}, \mathbf{n}, \mathbf{q})$. Merged images $(\mathbf{c}, \mathbf{f}, \mathbf{i}, \mathbf{l}, \mathbf{o}, \mathbf{r})$. Scale bar $=1000 \mu \mathrm{m}(\mathbf{a}-\mathbf{c}, \mathbf{g}-\mathbf{i}, \mathbf{m}-\mathbf{o})$. Scale bar $=100 \mu m(\mathbf{d}-\mathbf{f}, \mathbf{j}-\mathbf{I}, \mathbf{p}-\mathbf{r})$. Relative GFAP level (s). ${ }^{*} p<0.05$ compared with autograft, ${ }^{\#} p<0.05$ compared with $\mathrm{PCL}$

effects, such as donor site damage and nerve mismatch ${ }^{24}$. Therefore, there is a need for alternative biomimetic materials to reconstruct nerve connections physically and biologically. However, synthetic nerve conduits cannot effectively repair long-range nerve defects.

Carbon-based smart materials, such as CNTs, graphene, and NDs, have attracted widespread attention in recent years $^{25}$. CNTs have been used for drug or gene delivery.
They were reported to transport acetylcholine into the lysosome in neurons in an Alzheimer's disease model ${ }^{26}$. However, CNTs are very controversial in terms of their biocompatibility. Physicochemical characteristics greatly change the biological performance of CNTs. Raw CNT inhalation or peritoneal injection led to pathological changes in physiological functions and inflammatory responses $^{27}$. Graphene and its derivatives have been more 

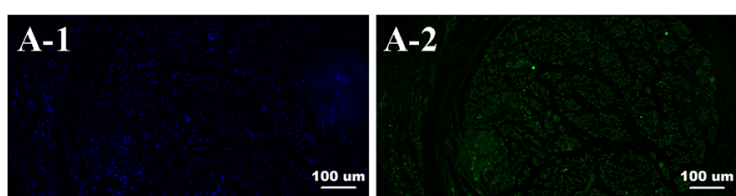

\section{A-3}
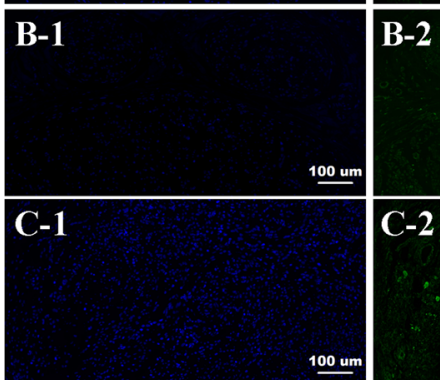

00 um

B-3
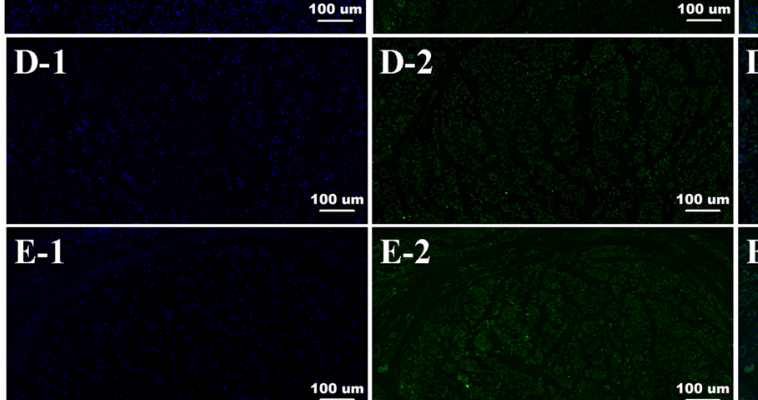

E-2

(1)

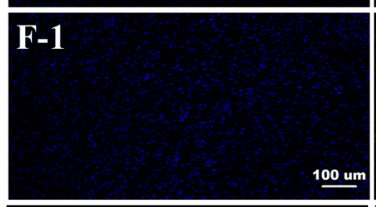

F-2
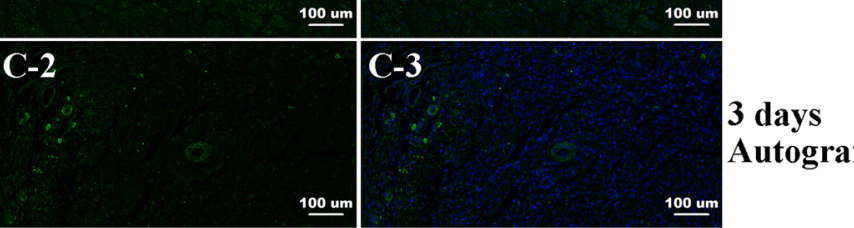

Autograft

\section{ND/PCL}

100 um

3 days

PCL

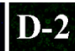

D-3

100 um

(1)

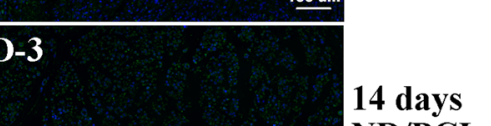

ND/PCL
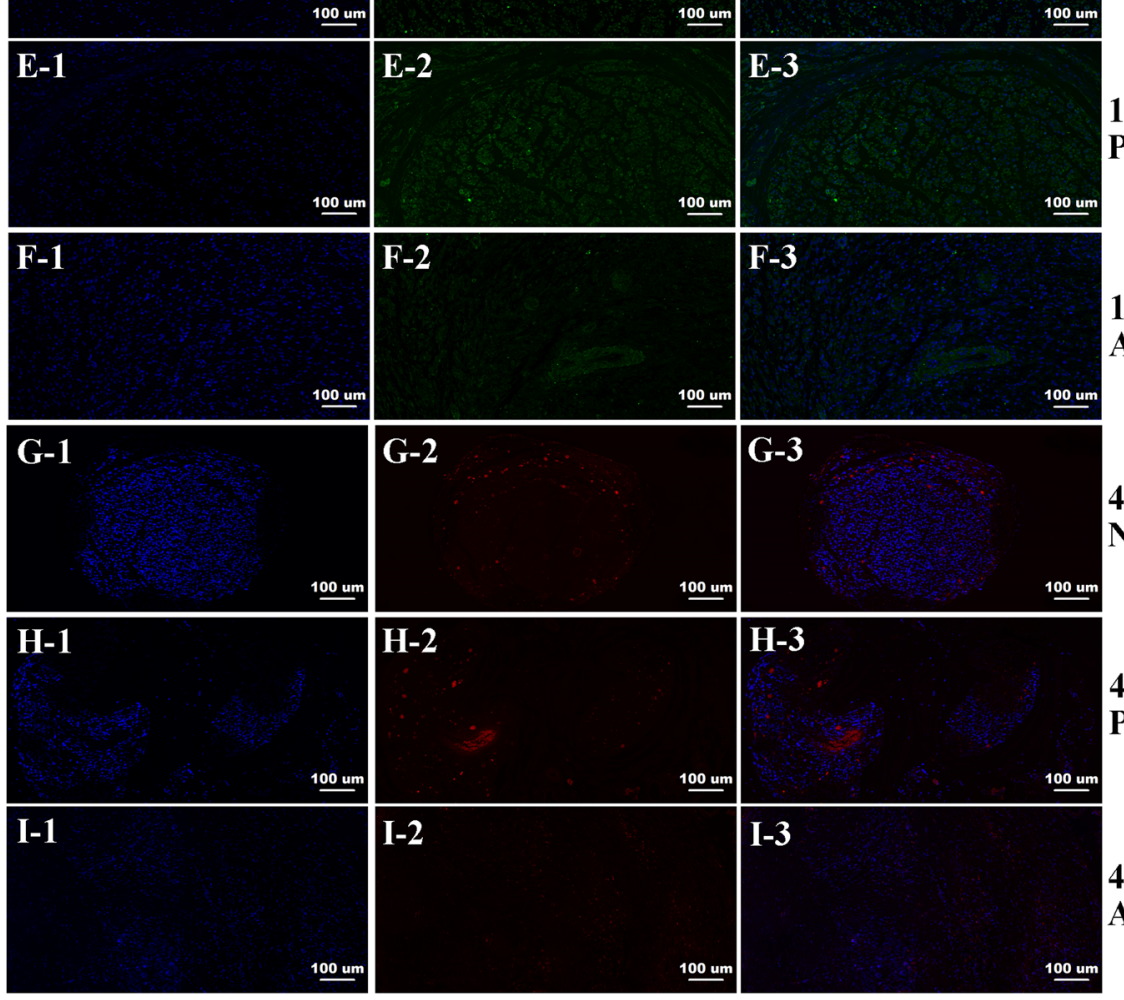

14 days

PCL

$100 \mathrm{um}$

F-3

oum

(n)

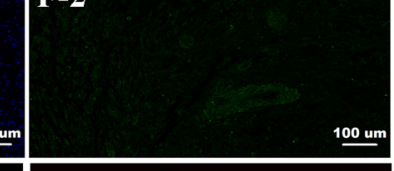

F-3

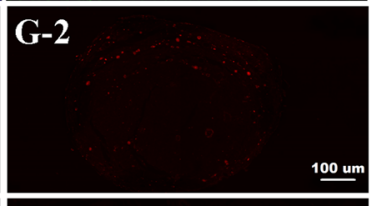

G-3

14 days

Autograft

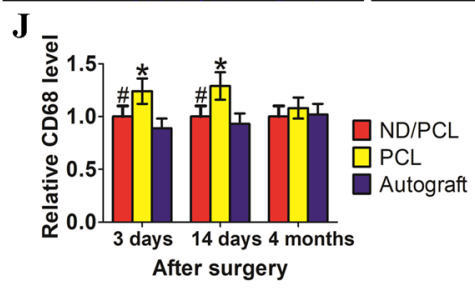

Fig. 10 Immunofluorescence assay of sciatic nerves using CD68 for macrophage state evaluation. Evaluation at 3 days postoperatively. Nanodiamond/polycaprolactone (ND/PCL) nerve bridge (a1-a3). PCL nerve bridge (b1-b3). Autograft (c1-c3). Evaluation at 14 days postoperatively. ND/PCL nerve bridge (d1-d3). PCL nerve bridge (e1-e3). Autograft $(\mathbf{f} \mathbf{1}-\mathbf{f} \mathbf{3})$. Evaluation at 4 months postoperatively. ND/PCL nerve bridge $(\mathbf{g} \mathbf{1}-\mathbf{g} \mathbf{3})$. PCL nerve bridge (h1-h3). Autograft (i1-i3). Nuclear staining (a1-i1). CD68 staining (a2-i2). Merged images (a3-i3). Scale bar $=100 \mu \mathrm{m}$. Relative CD68 level (j). ${ }^{*} p<0.05$ compared with autograft, ${ }^{\#} p<0.05$ compared with $\mathrm{PCL}$ 


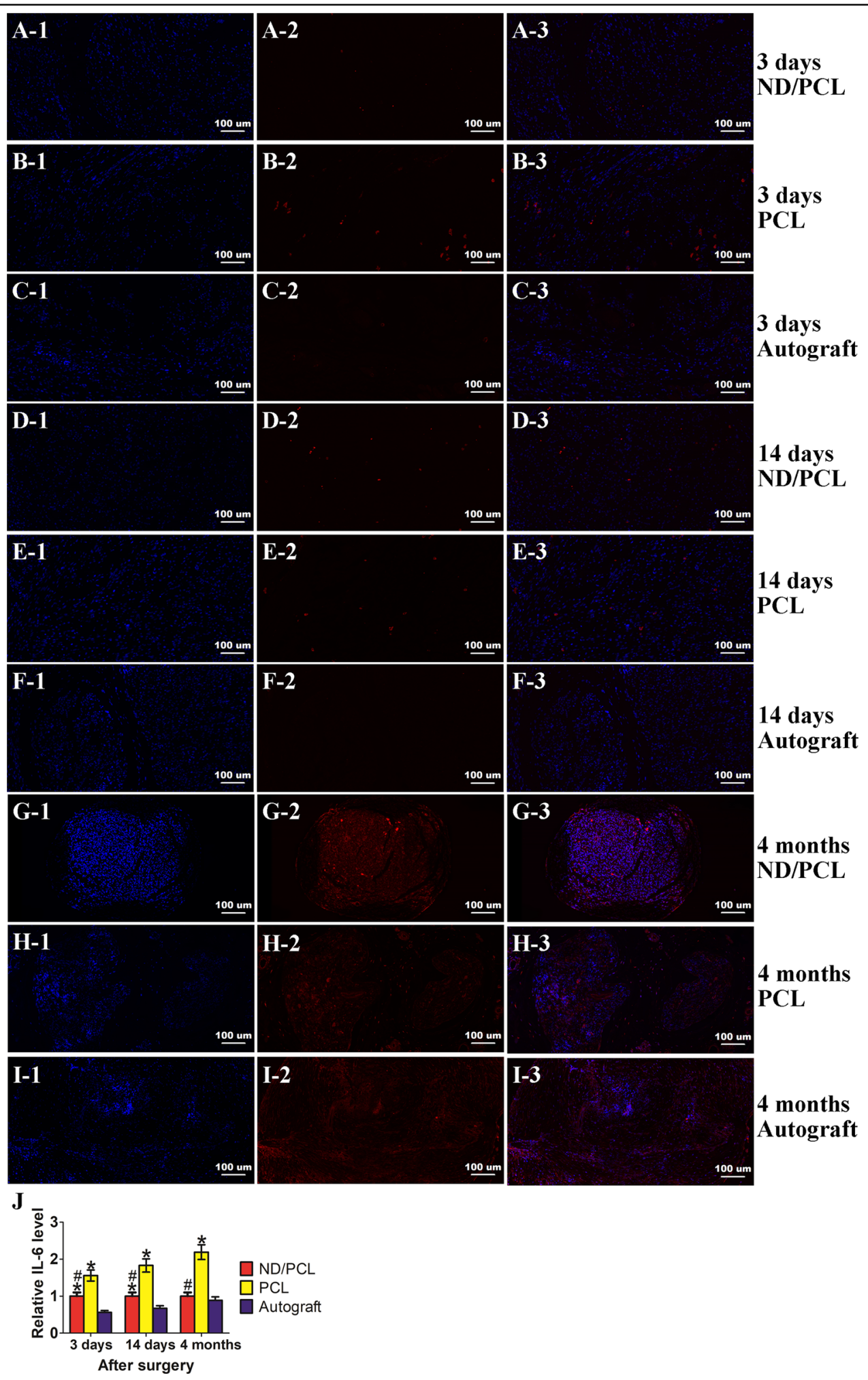

Fig. 11 Immunofluorescence assay of sciatic nerves using interleukin (IL)-6 for macrophage state evaluation. Evaluation at 3 days postoperatively. Nanodiamond/polycaprolactone (ND/PCL) nerve bridge (a1-a3). PCL nerve bridge (b1-b3). Autograft (c1-c3). Evaluation at 14 days postoperatively. ND/PCL nerve bridge (d1-d3). PCL nerve bridge (e1-e3). Autograft (f1-f3). Evaluation at 4 months postoperatively. ND/PCL nerve bridge (g1-g3). PCL nerve bridge (h1-h3). Autograft (i1-i3). Nuclear staining (a1-i1). IL-6 staining (a2-i2). Merged images (a3-i3). Scale bar= $100 \mu \mathrm{m}$. Relative IL-6 level (j). ${ }^{*} p<0.05$ compared with autograft, ${ }^{\#} p<0.05$ compared with $\mathrm{PCL}$ 


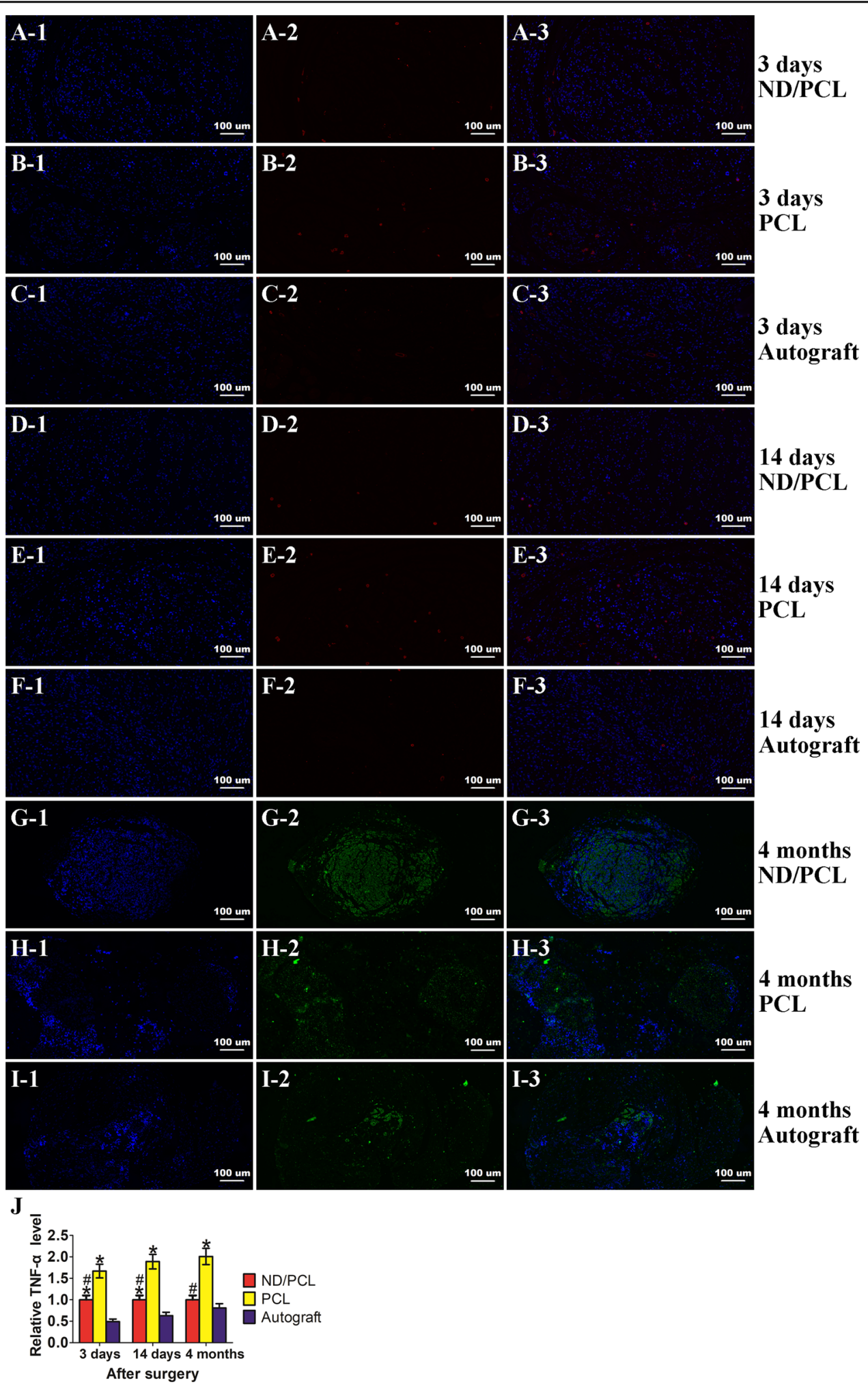

Fig. 12 Immunofluorescence assay of sciatic nerves using tumor necrosis factor (TNF)-a for macrophage state evaluation. Evaluation at 3 days postoperatively. Nanodiamond/polycaprolactone (ND/PCL) nerve bridge (a1-a3). PCL nerve bridge (b1-b3). Autograft (c1-c3). Evaluation at 14 days postoperatively. ND/PCL nerve bridge (d1-d3). PCL nerve bridge (e1-e3). Autograft (f1-f3). Evaluation at 4 months postoperatively. ND/PCL nerve bridge (g1-g3). PCL nerve bridge (h1-h3). Autograft (i1-i3). Nuclear staining (a1-i1). TNF-a staining (a2-i2). Merged images (a3-i3). Scale bar = $100 \mu \mathrm{mm}$. Relative TNF-a level (j). ${ }^{*} p<0.05$ compared with autograft, ${ }^{*} p<0.05$ compared with $\mathrm{PCL}$ 


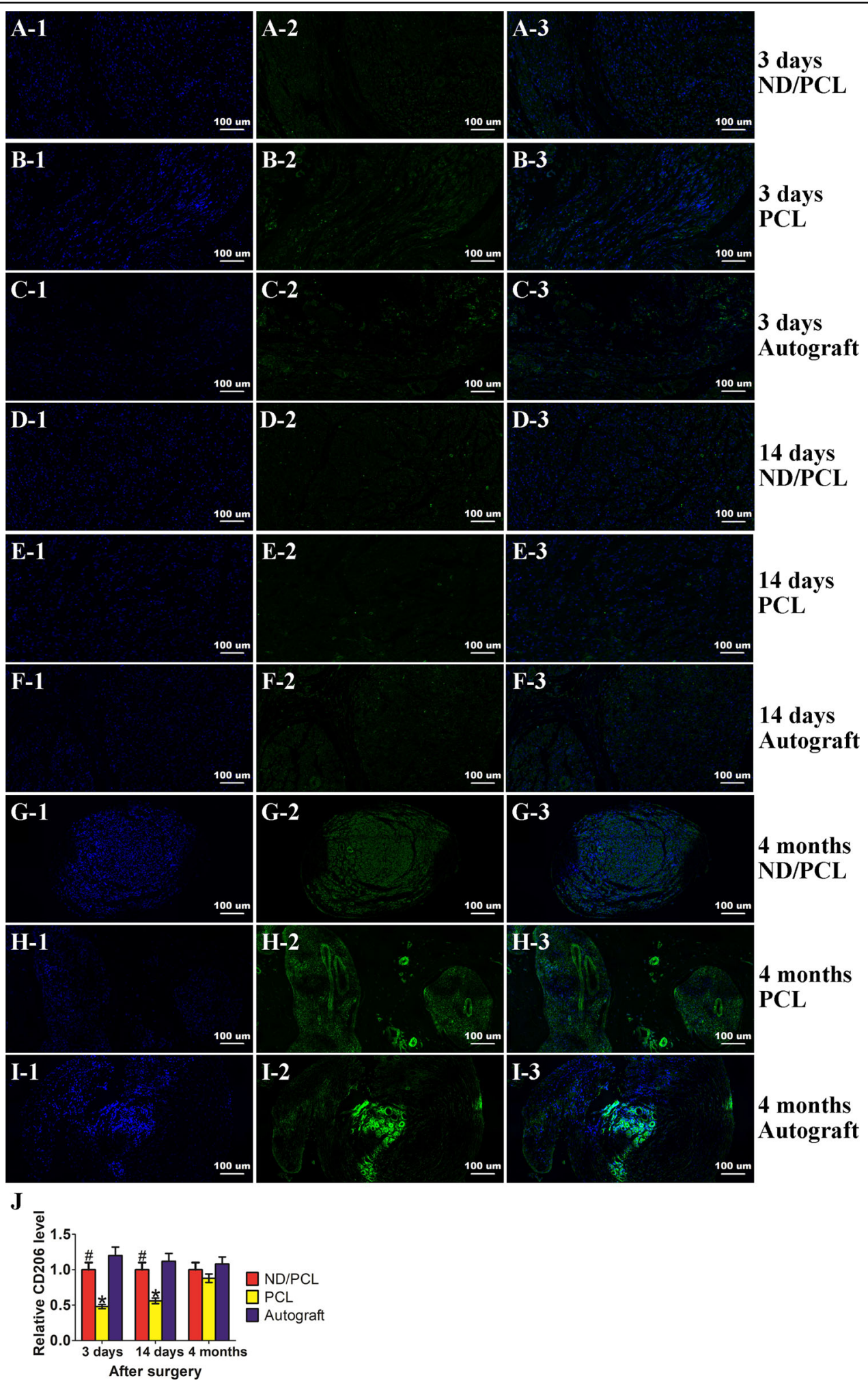

Fig. 13 Immunofluorescence assay of sciatic nerves using CD206 for macrophage state evaluation. Evaluation at 3 days postoperatively. Nanodiamond/polycaprolactone (ND/PCL) nerve bridge (a1-a3). PCL nerve bridge (b1-b3). Autograft (c1-c3). Evaluation at 14 days postoperatively. ND/PCL nerve bridge (d1-d3). PCL nerve bridge (e1-e3). Autograft (f1-f3). Evaluation at 4 months postoperatively. ND/PCL nerve bridge (g1-g3). PCL nerve bridge (h1-h3). Autograft (i1-i3). Nuclear staining (a1-i1). CD206 staining (a2-i2). Merged images (a3-i3). Scale bar $=100 \mu \mathrm{m}$. Relative CD206 level (j). ${ }^{*} p<0.05$ compared with autograft, ${ }^{\#} p<0.05$ compared with $\mathrm{PCL}$ 


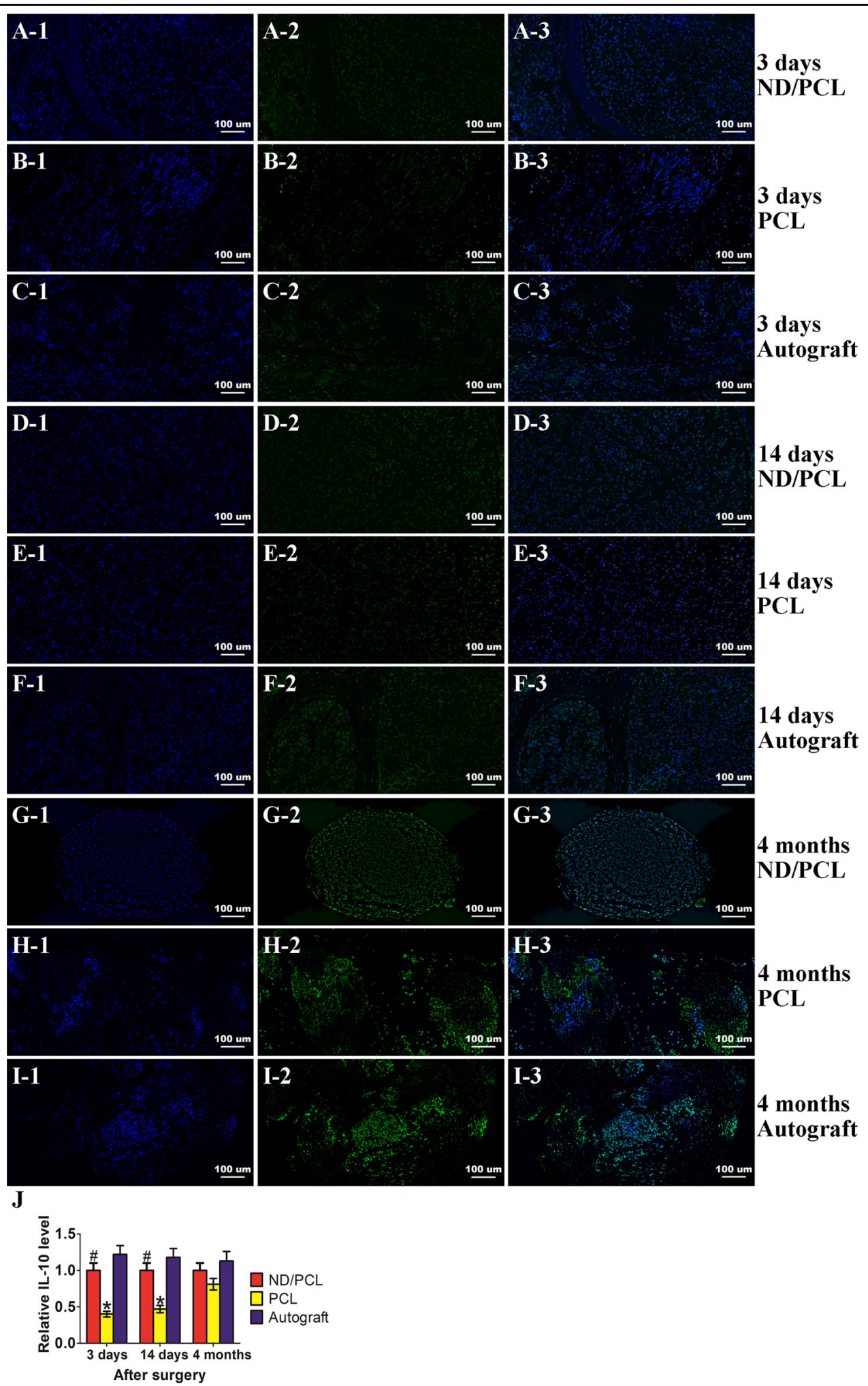

Fig. 14 Immunofluorescence assay of sciatic nerves using interleukin (IL)-10 for macrophage state evaluation. Evaluation at 3 days postoperatively. Nanodiamond/polycaprolactone (ND/PCL) nerve bridge (a1-a3). PCL nerve bridge (b1-b3). Autograft (c1-c3). Evaluation at 14 days postoperatively. ND/PCL nerve bridge (d1-d3). PCL nerve bridge (e1-e3). Autograft (f1-f3). Evaluation at 4 months postoperatively. ND/PCL nerve bridge (g1-g3). PCL nerve bridge (h1-h3). Autograft (i1-i3). Nuclear staining (a1-i1). IL-10 staining (a2-i2). Merged images (a3-i3). Scale bar= $100 \mu \mathrm{m}$. Relative IL-10 level (j). ${ }^{*} p<0.05$ compared with autograft, ${ }^{\#} p<0.05$ compared with PCL 


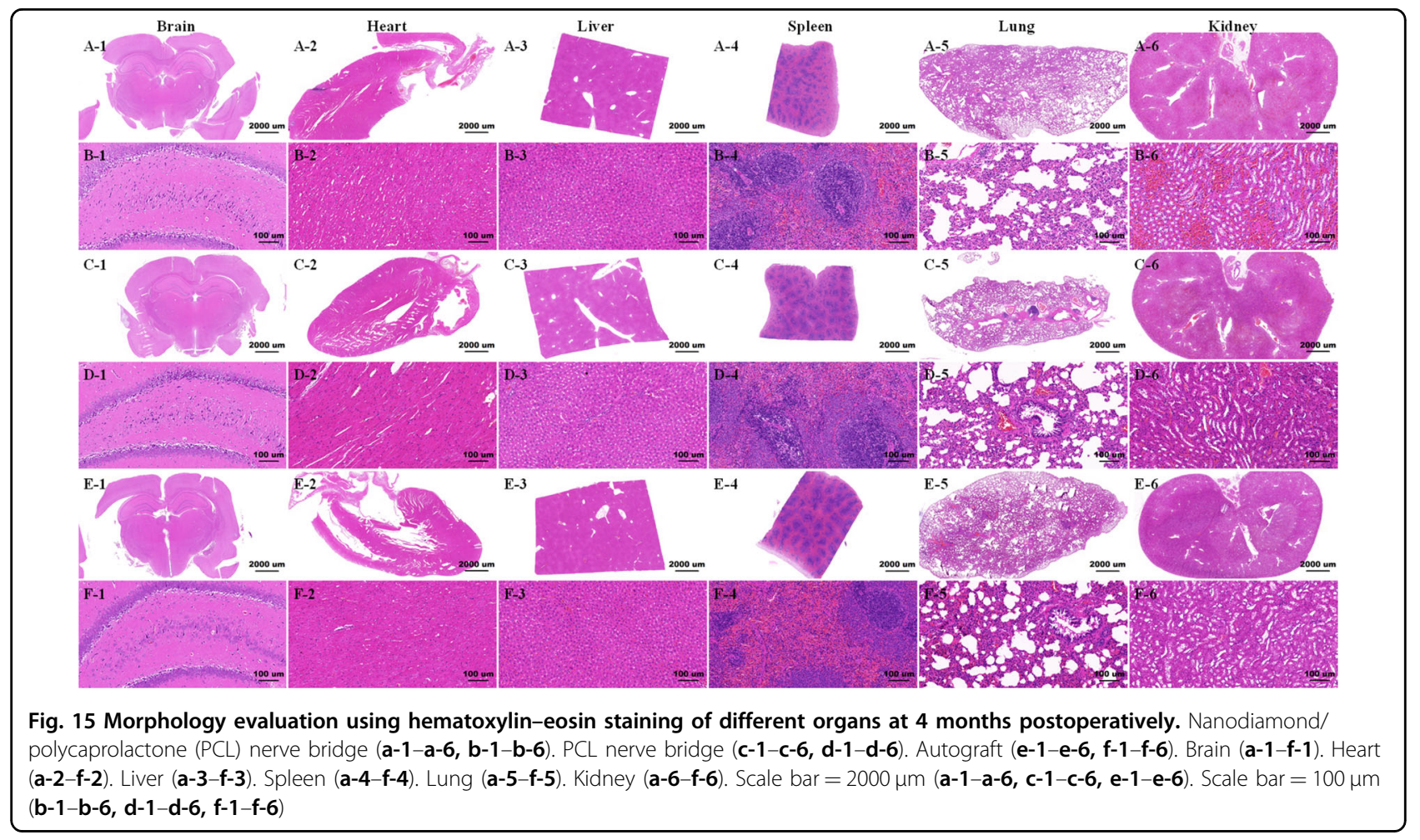

popular in tissue engineering than other members of the carbon family. Graphene toxicity is associated with its size, layers, shape, and dosage. Different nanoscales and layers of graphene sheets significantly alter the biosafety performance of graphene. Larger particles may become stuck in the airway or lungs, and smaller particles accumulate in hepatic tissues ${ }^{28}$. In our previous study, we found that $1 \%$ loading in PCL scaffolds was the maximum safe concentration for graphene or GO in in vitro assays.

The biocompatibility of ND is generally considered better than that of other carbon family members, such as graphene and CNT, and is therefore a good candidate for tissue regenerative scaffolds. In fact, there are still some controversies concerning the appropriate ND concentration for ideal cell viability in different cell lines. Previous reports indicated that $10 \% \mathrm{ND}$ was more beneficial to neuroblastoma SHSY5Y cell proliferation than $1.5 \%, 5 \%$, or $15 \% \mathrm{ND}^{14}$. Macrophages were not affected by ND at $50 \mu \mathrm{g} \mathrm{ml}^{-129}$. There is not a conclusion for these conflicting phenomena. Nanoparticle size may be a crucial factor. Therefore, purification procedures and various surface modifications are needed. Only when all the variables are constant, then we discuss ND biocompatibility in a thorough and comprehensive way. In this study, we fabricated ND/PCL nerve bridges using a concentric multilayer molding manufacturing process. The diameter of NDs was $10 \mathrm{~nm}$. We used staggered netshaped dotting for the surface modification. Under the present conditions, we were able to investigate ND-based nerve bridges in neuroengineering.

In this study, the ND nerve bridges were confirmed to induce a proliferative, adhesive, and glioprotective effect for SCs in vitro. All of these effects originated from the biocompatible nature of NDs. We further evaluated longterm in vivo performance in a $20-\mathrm{mm}$ sciatic nerve defect rat model. The ND bridges successfully repaired nerve defects after 4 months and rebuilt normal sciatic nerve function, morphology, and electrophysiological reactions. In addition, we investigated the potential apoptotic and toxic effects in the tested major rat organs. None of them displayed significant deformation or pathological changes in comparison with the autograft groups. These phenomena helped us confirm that NDs are very suitable for peripheral nerve tissue engineering due to its excellent biocompatibility. Some research has shown that dynamic cell-material interactions result in increased cell adhesion and better sensing of biological cues from scaffolds. The scaffolds were fabricated from nanofibers and enabled long-range cell-cell communication for better tissue repair ${ }^{30}$. We think this might partially explain the biocompatible, proliferative, and glioprotective effects of NDs.

In addition to biocompatibility, we also comprehensively analyzed the effect of NDs on macrophage polarization in this study to further investigate the reasons behind their glioprotective and regenerative capability. 
Macrophages have a well-known function, removing myelin debris during the early period after peripheral nerve injuries. This process usually ends within $48 \mathrm{~h}$ in a physical atmosphere ${ }^{31}$. However, macrophages recruit chemokines persistently and cause lasting and chronic inflammatory responses that block functional nerve regrowth. M1 macrophages (proinflammatory type) are responsible for these effects. In contrast, the polarization of M1 into M2 macrophages (anti-inflammatory type) can efficiently result in nerve proliferation and regeneration. The detection of M2 macrophages is usually concentrated in the distal end of nerve stumps. M2 macrophages are the pro-healing type and improve tissue regeneration by secreting anti-inflammatory growth factors and cytokines, which contribute to cell adhesion, proliferation, and nutritional milieu restoration ${ }^{32}$. IL-4 release from the nerve conduit stimulated increased number of macrophages that were positive for M2 markers, such as CD163 and CD206, and promoted nerve fiber elongation inside the conduit $^{33}$. Another study also showed that the collagen-induced M2 increase was associated with enhanced structural and functional recovery after sciatic nerve transection injury ${ }^{34}$. Therefore, we may reverse lasting inflammation and regulate the immune milieu by applying nanomaterials that are capable of inducing macrophage polarization from M1 to M2 in a timely manner. In this study, we evaluated this aspect both in vitro and in vivo. SCs and regenerated nerves in ND bridges showed lower proinflammatory markers, such as TNF- $\alpha$ and IL-6, and higher anti-inflammatory cues, such as CD206 and IL-10, than their PCL counterparts. Previous reports also showed that NDs at $10-20 \mu \mathrm{g} \mathrm{ml}^{-1}$ could stimulate transforming growth factor $\beta$ and granulocyte-macrophage colony stimulating factor activation and decrease IL- $1 \alpha$ and TNF- $\alpha$ expression, thus regulating the immune response in the microenvironment $^{35}$. In addition, we analyzed STAT-3 (M2 phenotyperelated signaling protein) and NF- $\mathrm{KB}$ (M1 phenotyperelated signaling protein) expression ${ }^{36}$. These results also confirmed that the ND/PCL nerve bridges polarized macrophages into the M2 type via the Janus-activated kinase-STAT signaling pathway. Additionally, NDs led to prominent angiogenesis and muscle reinnervation in the regenerative process, which contributed to ideal nerve regeneration jointly. Low inflammatory levels stimulate angiogenesis and thus offer nutrients to effector muscles $^{37}$. Therefore, we believe that the biocompatible, proliferative, and glioprotective ability of ND nerve bridges also partially originates from inflammatory control, macrophage regulation, and polarization during long-term peripheral nerve regeneration. Cell-cell interaction enhancement and macrophage polarization are two main factors that should explain the regenerative capability of ND nerve bridges in repairing 20-mm sciatic nerve defects within 4 months.

There are some novelties of this study. Above all, this is the first time that NDs have been investigated for their glioprotective effects in the animal model. In previous studies, NDs were given to animals through intravenous, subcutaneous, inhalation, and oral pathways ${ }^{38}$. We thoroughly demonstrated that sciatic nerve recovery in ND/ PCL conduits was comparable to that in autologous grafts. In addition, we found that the GFAP expression level in the brain in the ND/PCL group was also significantly higher than that in its PCL counterpart. The consistency with the peripheral nerve indicated that central nervous system function was regulated and improved by ND application as well. Although the detailed mechanism is unclear, this inspires us to investigate the central and peripheral connection in peripheral nerve repair in the future.

Second, this is the first time that carbon-based nanomaterials have been used for repairing $20-\mathrm{mm}$ peripheral nerve defects. We used single-layered and multilayered graphene as well as GO to fabricate graphene-based nerve conduits. Their effects on nerve regeneration were investigated comprehensively at 18 weeks after surgery in vivo. However, the defect was $15 \mathrm{~mm}$, shorter than the $20 \mathrm{~mm}$ defect used in this study. ND/PCL conduits could basically repair severe defects.

Third, this is the first time that concentric manufacturing has been used for biomimetic nerve conduits. In this study, we combined multilayered spraying and gradient dotting to facilitate a more biocompatible and biodegradable nerve bridge. Concentric architecture provided an elastic multilayered wall with higher flexibility and stability. Staggered microporous surfaces prevented fibroblasts from entering the conduit lumen better than our previous products. In addition, the concentric manufacturing technique allowed steady ND release. This approach has many advantages, such as selective targeting, stability of drugs in physiological environments, and lower side effects of therapeutic agents ${ }^{39}$. Moreover, a multilayered architecture provides cell-cell interactions from all sides instead of only horizontal and vertical intercellular connections in 2D scaffolds. Biomimetic nerve bridges lead nerve cells to extend from near to distant and facilitate cell adhesion, spreading, proliferation, migration, and differentiation ${ }^{40}$.

Finally, this is also the first time that NDs have been confirmed to regulate macrophage polarization in severe sciatic nerve defects. NDs recruited macrophages and induced the M1 type into the M2 type. At 4 months after surgery, the regenerated nerves in the ND/PCL conduit still exhibited significantly higher CD206 and IL-10 expression levels than those in the other groups. 
Successful manipulation and recruitment of macrophages was one of the main factors in ND-dependent nerve repair.

\section{Conclusion}

We evaluated the biocompatibility of NDs thoroughly in long-term peripheral nerve regeneration. Compared with other carbon-based materials such as graphene and CNT, NDs barely harm organs and tissues in the living body. In addition, NDs also regulate the immune milieu by inducing macrophage polarization. The ND/PCL nerve bridge not only reconstructs neural continuity but also restores the biocompatible microenvironment for peripheral nerve regeneration. These prominent advantages guarantee the clinical translation of ND-based biomaterials in neuroengineering.

\section{Acknowledgements}

This study was supported by the Projects of National Science Foundation of China (Grant Nos. 81830076 and 81672146), the Projects of Nature Science Foundation of Shanghai, China (Grant No. 19ZR1439200), the SUMHS Seed Foundation Project (Grant No. HMSF-16-21-010), and the Science and Technology Development Foundation of Pudong New District, Shanghai, China (Grant Nos. PKJ2016-Y55 and PWZxq2017-03). This study was also partly sponsored by the Interdisciplinary Program of Shanghai Jiao Tong University (Nos. YG2017MS22 and YG2017QN56) and the Translational Medicine Program of Shanghai Jiao Tong University (No. ZH2018QNA56). The study was also partly sponsored by the Program of Shanghai Sixth People's Hospital East Campus Foundation (No. 2019YY001) and the Program of Shanghai Sixth People's Hospital Foundation (No. LY2Y0272). We appreciate assistance from the faculty of the Instrumental Analysis Center (IAC) of Shanghai Jiao Tong University.

\section{Author details}

${ }^{1}$ Department of Orthopedics, Shanghai Jiao Tong University Affiliated Sixth People's Hospital, 200233 Shanghai, China. ${ }^{2}$ Engineering Research Center of Cell \& Therapeutic Antibody, Ministry of Education, and School of Pharmacy, Shanghai Jiao Tong University, 200240 Shanghai, China. ${ }^{3}$ Shanghai Sixth People's Hospital East Affiliated to Shanghai University of Medicine \& Health Sciences, 201306 Shanghai, China

\section{Author contributions}

W.-E.Y. conceived the initial idea and the conceptualization. W.-E.Y. and C.F. designed the study and participated in the data extraction and analysis. Y.Q., Y. C., and Y.O. participated in study design, searched databases, assessed studies, and drafted the manuscript. Y.Q. wrote and revised the manuscript. All authors read and approved the final manuscript.

\section{Conflict of interest}

The authors declare that they have no conflict of interest.

\section{Publisher's note}

Springer Nature remains neutral with regard to jurisdictional claims in published maps and institutional affiliations.

Supplementary information is available for this paper at https://doi.org/ 10.1038/s41427-019-0136-8.

Received: 28 November 2018 Revised: 12 May 2019 Accepted: 17 May 2019. Published online: 19 July 2019

\section{References}

1. Qian, Y. et al. Asymmetrical 3D nanoceria channel for severe neurological defect regeneration. iscience 12, 216-231 (2019).

2. Qian, Y. et al. 3D melatonin nerve scaffold reduces oxidative stress and inflammation and increases autophagy in peripheral nerve regeneration. J. Pineal Res. 65, e12516 (2018).

3. Riccio, M., Marchesini, A., Pugliese, P. \& De Francesco, F. Nerve repair and regeneration: biological tubulization limits and future perspectives. J. Cell. Physiol. 234, 3362-3375 (2019).

4. Saito, N. et al. Safe clinical use of carbon nanotubes as innovative biomaterials. Chem. Rev. 114, 6040-6079 (2014).

5. Oprych, K. M., Whitby, R. L. D., Mikhalovsky, S. V., Tomlins, P. \& Adu, J. Repairing peripheral nerves: is there a role for carbon nanotubes? Adv. Healthc. Mater. $\mathbf{5}$ 1253-1271 (2016).

6. Chatterjee, N., Eom, H. J. \& Choi, J. A systems toxicology approach to the surface functionality control of graphene-cell interactions. Biomaterials $\mathbf{3 5}$, 1109-1127 (2014).

7. Nistor, P. A., May, P. W., Tamagnini, F., Randall, A. D. \& Caldwell, M. A. Long-term culture of pluripotent stem-cell-derived human neurons on diamond-a substrate for neurodegeneration research and therapy. Biomaterials 61, 139-149 (2015).

8. Ahn, H. S. et al. Carbon-nanotube-interfaced glass fiber scaffold for regeneration of transected sciatic nerve. Acta Biomater. 13, 324-334 (2015).

9. Salehi, M. et al. Sciatic nerve regeneration by transplantation of Schwann cells via erythropoietin controlled-releasing polylactic acid/multiwalled carbon nanotubes/gelatin nanofibrils neural guidance conduit. J. Biomed. Mater. Res. B Appl. Biomater. 106, 1463-1476 (2018).

10. Qian, Y. et al. An integrated multi-layer 3D-fabrication of PDA/RGD coated graphene loaded PCL nanoscaffold for peripheral nerve restoration. Nat. Commun. 9, 323 (2018).

11. Qian, Y. et al. 3D fabrication with integration molding of a graphene oxide/ polycaprolactone nanoscaffold for neurite regeneration and angiogenesis. Adv. Sci. 5, 1700499 (2018)

12. Lee, S. K. et al. 3D-networked carbon nanotube/diamond core-shell nanowires for enhanced electrochemical performance. NPG Asia Mater. 6, e115 (2014).

13. Yu, S. J., Kang, M. W., Chang, H. C., Chen, K. M. \& Yu, Y. C. Bright fluorescent nanodiamonds: no photobleaching and low cytotoxicity. J. Am. Chem. Soc. 127, 17604-17605 (2005).

14. Aida, V. et al. Impact of diamond nanoparticles on neural cells. Mol. Cell. Probes 29, 25-30 (2015).

15. Schrand, A. M. et al. Are diamond nanoparticles cytotoxic? J. Phys. Chem. B 111, 2-7 (2007)

16. Hopper, A. P. et al. Amine functionalized nanodiamond promotes cellular adhesion, proliferation and neurite outgrowth. Biomed. Mater. 9, 045009 (2014).

17. Thalhammer, A., Edgington, R. J., Cingolani, L. A., Schoepfer, R. \& Jackman, R. B. The use of nanodiamond monolayer coatings to promote the formation of functional neuronal networks. Biomaterials 31, 2097-2104 (2010).

18. Pentecost, A. E., Witherel, C. E., Gogotsi, Y. \& Spiller, K. L. Anti-inflammatory effects of octadecylamine-functionalized nanodiamond on primary human macrophages. Biomater. Sci. 5, 2131-2143 (2017).

19. Mokarram, N., Merchant, A., Mukhatyar, V., Patel, G. \& Bellamkonda, R. V. Effect of modulating macrophage phenotype on peripheral nerve repair. Biomaterials 33, 8793-8801 (2012).

20. Nadeau, S. et al. Functional recovery after peripheral nerve injury is dependent on the pro-inflammatory cytokines $\mathrm{IL}-1 \beta$ and TNF: implications for neuropathic pain. J. Neurosci. 31, 12533-12542 (2011).

21. Lu, T. S., Yiao, S. Y., Lim, K., Jensen, R. V. \& Hsiao, L. L. Interpretation of biological and mechanical variations between the Lowry versus Bradford method for protein quantification. N. Am. J. Med. Sci. 2, 325-328 (2010).

22. Qian, Y. et al. 3D manufacture of gold nanocomposite channels facilitates neural differentiation and regeneration. Adv. Funct. Mater. 28, 1707077 (2018).

23. Frost, S. J. et al. Gecko-inspired chitosan adhesive for tissue repair. NPG Asia Mater. 8, e280 (2016).

24. Afshari, A. et al. Assessment of the effect of autograft orientation on peripheral nerve regeneration using diffusion tensor imaging. Ann. Plast. Surg. $\mathbf{8 0}$ 384-390 (2018).

25. Monaco, A. M. \& Giugliano, M. Carbon-based smart nanomaterials in biomedicine and neuroengineering. Beilstein J. Nanotechnol. 5, 1849-1863 (2014). 
26. Yang, Z. et al. Pharmacological and toxicological target organelles and safe use of single-walled carbon nanotubes as drug carriers in treating Alzheimer disease. Nanomedicine 6, 427-441 (2010).

27. Poland, C. A. et al. Carbon nanotubes introduced into the abdominal cavity of mice show asbestos-like pathogenicity in a pilot study. Nat. Nanotechnol. 3, 423-428 (2008)

28. Liu, J. H. et al. Effect of size and dose on the biodistribution of graphene oxide in mice. Nanomedicine 7, 1801-1812 (2012).

29. Thomas, V., Halloran, B. A., Ambalavanan, N., Catledge, S. A. \& Vohra, Y. K. In vitro studies on the effect of particle size on macrophage responses to nanodiamond wear debris. Acta Biomater. 8, 1939-1947 (2012).

30. Chan, C. E. \& Odde, D. J. Traction dynamics of filopodia on compliant substrates. Science 322, 1687-1691 (2008).

31. Klein, D. \& Martini, R. Myelin and macrophages in the PNS: an intimate relationship in trauma and disease. Brain Res. 1641, 130-138 (2016).

32. Das, A. et al. Monocyte and macrophage plasticity in tissue repair and regeneration. Am. J. Pathol. 185, 2596-2606 (2015).

33. Zigmond, R. E. \& Echevarria, F. D. Macrophage biology in the peripheral nervous system after injury. Prog. Neurobiol. 173, 102-121 (2019).
34. LV, D., Zhou, L., Zheng, X. \& Hu, Y. Sustained release of collagen VI potentiates sciatic nerve regeneration by modulating macrophage phenotype. Eur. J. Neurosci. 45, 1258-1267 (2017).

35. Shkurupy, V. A., Arkhipov, S. A., Neshchadim, D. V., Akhramenko, E. S. \& Troitskii, A. V. In Vitro Effects of Nanosized Diamond Particles on Macrophages. Bull. Exp. Biol. Med. 158, 500-503 (2015).

36. Peng, Q. et al. Interleukin 29 inhibits RANKL-induced osteoclastogenesis via activation of JNK and STAT, and inhibition of NF-KB and NFATC1. Cytokine 113, 144-154 (2019).

37. Szade, A., Grochot-Przeczek, A., Florczyk, U., Jozkowicz, A. \& Dulak, J. Cellular and molecular mechanisms of inflammation-induced angiogenesis. IUBMB Life 67, 145-159 (2015).

38. van der Laan, K., Hasani, M., Zheng, T. \& Schirhagl, R. Nanodiamonds for In Vivo Applications. Small 14, e1703838 (2018).

39. Whitlow, J. Pacelli, S. \& Paul, A. Multifunctional nanodiamonds in regenerative medicine: Recent advances and future directions. J. Control. Release 261, 62-86 (2017).

40. Schwartz, M. A. \& Chen, C. S. Cell biology. Deconstructing dimensionality. Science 339, 402-404 (2013). 